\title{
A PROBLEM ABOUT MAHLER FUNCTIONS
}

\author{
by
}

\author{
Boris Adamczewski \& Jason P. Bell
}

In memory of Alf van der Poorten

\begin{abstract}
Let $K$ be a field of characteristic zero and $k$ and $l$ be two multiplicatively independent positive integers. We prove the following result that was conjectured by Loxton and van der Poorten during the Eighties: a power series $F(z) \in K[[z]]$ satisfies both a $k$ - and a $l$-Mahler type functional equation if and only if it is a rational function.
\end{abstract}

\section{Contents}

1. Introduction............................... 2

2. Connection with finite automata and Cobham's theorem. . 7

3. Sketch of proof of Theorem $1.1 \ldots \ldots \ldots \ldots \ldots \ldots \ldots \ldots .6$

4. Preliminary reduction for the form of Mahler equations. . 10

5. Reduction to the number field case ................ 12

6. Further reductions for the form of Mahler equations..... 16

7. Links with automatic and regular power series......... 19

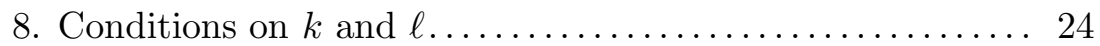

9. Elimination of singularities at roots of unity........... 26

10. Existence of prime ideals with special properties....... 42

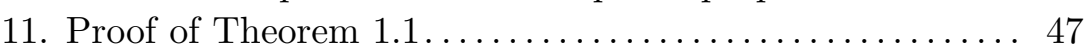

References.............................. 50

The first author was supported by the project Hamot, ANR 2010 BLAN-0115-01. The second author was supported by NSERC grant 31-611456. 


\section{Introduction}

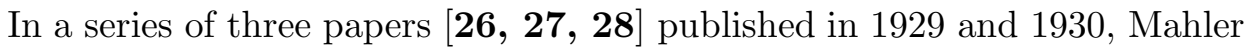
initiated a totally new direction in transcendence theory. Mahler's method, a term coined much later by Loxton and van der Poorten, aims at proving transcendence and algebraic independence of values at algebraic points of locally analytic functions satisfying certain type of functional equations. In its original form, it concerns equations of the form

$$
F\left(z^{k}\right)=R(z, F(z)),
$$

where $R(z, x)$ denotes a bivariate rational function with coefficients in a number field. For instance, using the fact that $F(z)=\sum_{n=0}^{\infty} z^{2^{n}}$ satisfies the basic functional equation

$$
F\left(z^{2}\right)=F(z)-z
$$

Mahler was able to prove that $F(\alpha)$ is a transcendental number for every algebraic number $\alpha$ with $0<|\alpha|<1$. As observed by Mahler himself, his approach allows one to deal with functions of several variables and systems of functional equations as well. It also leads to algebraic independence results, transcendence measures, measures of algebraic independence, and so forth. Mahler's method was later developed by various authors, including Becker, Kubota, Loxton and van der Poorten, Masser, Nishioka, Töpfer, among others. For classical aspects of Mahler's theory, we refer the reader to the monograph of Ku. Nishioka [34] and the reference therein. However, a major deficiency of Mahler's method is that, contrary to Siegel E- and G-functions, there is not a single classical transcendental constant that is known to be the value at an algebraic point of an analytic function solution to a Mahler-type functional equation ${ }^{(*)}$. This may explain why it was somewhat neglected for almost fifty years.

At the beginning of the Eighties, Mahler's method really took on a new significance after Mendès France popularized the fact that some Mahler-type systems of functional equations naturally arise in the study of automata theory (see for instance [30]). Though already noticed in 1968 by Cobham [9], this connection remained relatively unknown at that time, probably because Cobham's work was never published in an academic journal. Cobham claimed that Mahler's method has the following nice consequence for the HartmanisStearns problem about the computational complexity of algebraic irrational

*. A remarkable discovery of Denis, which deserves to be better understood, is that Mahler's method can be also applied to prove transcendence and algebraic independence results involving periods of $t$-modules which are variants of the more classical periods of abelian varieties, in the framework of the arithmetic of function fields of positive characteristic. For a detailed discussion on this topic, we refer the reader to the recent survey by Pellarin [36], see also $[35]$. 
real numbers $[\mathbf{1 8}]$ : the expansion of an algebraic irrational number in an integer base cannot be generated by a finite automaton. His idea was to derive this result by applying Mahler's method to systems of functional equations of the form

$$
\left(\begin{array}{c}
F_{1}\left(z^{k}\right) \\
\vdots \\
F_{n}\left(z^{k}\right)
\end{array}\right)=A(z)\left(\begin{array}{c}
F_{1}(z) \\
\vdots \\
F_{n}(z)
\end{array}\right)+B(z)
$$

where $A(z)$ is an $n \times n$ matrix and $B(z)$ is an $n$-dimensional vector, both having entries that are rational functions with algebraic coefficients. Though Cobham's conjecture is now proved in [1] by mean of a completely different approach, it still remains a challenging problem to complete the proof he envisaged. In this direction, a great deal of work has been done by Loxton and van der Poorten $[\mathbf{2 4}, \mathbf{2 5}]$ and a particular attention was then paid to systems of functional equations as in (1.2) (see for instance $[\mathbf{3 1}, \mathbf{3 2}, \mathbf{3 4}, \mathbf{7}]$ ).

Let $K$ be field. We observe that a power series $F(z) \in K[[z]]$ is a component of a vector satisfying a system of functional equations of the form $(1.2)^{(*)}$ if and only if the family

$$
1, F(z), F\left(z^{k}\right), F\left(z^{k^{2}}\right), \ldots
$$

is linearly dependent over the field $K(z)$, that is, if there exist a natural number $n$ and polynomials $A(z), P_{0}(z), \ldots, P_{n}(z) \in K[z]$, not all of which are zero, such that

$$
A(z)+\sum_{i=0}^{n} P_{i}(z) F\left(z^{k^{i}}\right)=0 .
$$

Following Loxton and van der Poorten [25], we say that a power series $F(z) \in$ $K[[z]]$ is a $k$-Mahler function, or for short is $k$-Mahler, if it satisfies a functional equation of the form (1.3).

Beyond transcendence, Mahler's method and automata theory, it is worth mentioning that Mahler functions naturally occur as generating functions in various other topics such as combinatorics of partitions, numeration and the analysis of algorithms (see $[\mathbf{1 2}]$ and the references therein and also dozens of examples in $[\mathbf{5 , 6}]$ and $[\mathbf{1 6}$, Chapter 14]). A specially intriguing appearance of Mahler functions is related to the study of Siegel $G$-functions and in particular of diagonals of rational functions ${ }^{(*)}$. Though no general result confirms this claim, one observes that many generating series associated with the $p$-adic

*. We assume here that the entries of $A(z)$ and $B(z)$ are in $K(z)$.

*. See for instance [3] for a discussion of the links between diagonals of rational functions with algebraic coefficients and $G$-functions. 
valuation of the coefficients of $G$-functions with rational coefficients turn out to be $p$-Mahler functions.

As a simple illustration, we give the following example. Let us consider the algebraic function

$$
\mathfrak{f}(z):=\frac{1}{(1-z) \sqrt{1-4 z}}=\sum_{n=0}^{\infty} \sum_{k=0}^{n}\left(\begin{array}{c}
2 k \\
k
\end{array}\right) z^{n}
$$

and define the sequence

$$
a(n):=\nu_{3}\left(\sum_{k=0}^{n}\left(\begin{array}{c}
2 k \\
k
\end{array}\right)\right)
$$

where $\nu_{3}$ denotes the 3 -adic valuation. We claim that the function

$$
\mathfrak{f}_{1}(z):=\sum_{n \geq 0} a(n) z^{n} \in \mathbb{Q}[[z]]
$$

is a 3-Mahler function. This actually comes from the following nice equality

$$
\nu_{3}\left(\sum_{k=0}^{n}\left(\begin{array}{c}
2 k \\
k
\end{array}\right)\right)=\nu_{3}\left(n^{2}\left(\begin{array}{c}
2 n \\
n
\end{array}\right)\right),
$$

independently proved by Allouche and Shallit in 1989 (unpublished) and by Zagier [42]. Indeed, setting $\mathfrak{f}_{2}(z):=\sum_{n \geq 0} a(3 n) z^{n}$ and $\mathfrak{f}_{3}(z):=\sum_{n \geq 0} f(3 n+$ 1) $z^{n}$, we infer from Equality (1.4) that

$$
\left(\begin{array}{c}
\mathfrak{f}_{1}\left(z^{3}\right) \\
\mathfrak{f}_{2}\left(z^{3}\right) \\
\mathfrak{f}_{3}\left(z^{3}\right)
\end{array}\right)=A(z)\left(\begin{array}{c}
\mathfrak{f}_{1}(z) \\
\mathfrak{f}_{2}(z) \\
\mathfrak{f}_{3}(z)
\end{array}\right)+B(z)
$$

with

$$
A(z):=\frac{1}{z^{3}\left(1+z+z^{2}\right)}\left(\begin{array}{ccc}
z\left(1+z+z^{2}\right) & -z^{2} & -z \\
0 & z^{2}(1+z) & -z^{4} \\
0 & -z^{2} & z^{2}(1+z)
\end{array}\right)
$$


and

$$
B(z):=\frac{1}{z^{3}\left(1+z+z^{2}\right)}\left(\begin{array}{c}
\frac{z\left(2 z^{2}-1\right)}{z-1} \\
-\frac{z^{4}}{z-1} \\
\frac{z^{2}(1+z)}{z-1}
\end{array}\right) .
$$

A simple computation then gives the relation

$$
a_{0}(z)+a_{1}(z) \mathfrak{f}_{1}(x)+a_{2}(z) \mathfrak{f}_{1}\left(z^{3}\right)+a_{3}(z) \mathfrak{f}_{1}\left(z^{9}\right)+a_{4}(z) \mathfrak{f}_{1}\left(z^{27}\right)=0,
$$

where

$$
\begin{aligned}
a_{0}(z):= & z+2 z^{2}-z^{3}+z^{4}+3 z^{5}-z^{7}+3 z^{8}+z^{9}-z^{11}+3 z^{12}-2 z^{14} \\
& -z^{15}+2 z^{16}-2 z^{17}-2 z^{18}+2 z^{21} \\
a_{1}(z):= & -1-z^{4}-z^{8}+z^{9}+z^{13}+z^{17} \\
a_{2}(z):= & 1+z+z^{2}+z^{3}+z^{4}+z^{5}+z^{6}+z^{7}+z^{8}-z^{13}-z^{14}-z^{15}-z^{16} \\
& -z^{17}-z^{18}-z^{19}-z^{20}-z^{21} \\
a_{3}(z):= & -z^{3}-z^{6}-z^{7}-z^{9}-z^{10}-z^{11}-z^{13}-z^{14}+z^{16}-z^{17}+z^{19} \\
& +z^{20}+z^{22}+z^{23}+z^{24}+z^{26}+z^{27}+z^{30} \\
a_{4}(z):= & z^{21}-z^{48}
\end{aligned}
$$

Of course, considering the Hadamard product (denoted by $\odot$ below) of several algebraic functions would lead to similar examples associated with transcendental $G$-functions. For instance, the elliptic integral

$$
\mathfrak{g}(z):=\frac{2}{\pi} \int_{0}^{\pi / 2} \frac{d \theta}{\sqrt{1-16 z \sin ^{2} \theta}}=\frac{1}{\sqrt{1-4 z}} \odot \frac{1}{\sqrt{1-4 z}}=\sum_{n=0}^{\infty}\left(\begin{array}{c}
2 n \\
n
\end{array}\right)^{2} z^{n}
$$

is a transcendental $G$-function and it is not hard to see that, for every prime $p$,

$$
\mathfrak{g}_{p}(z):=\sum_{n=0}^{\infty} \nu_{p}\left(\left(\begin{array}{c}
2 n \\
n
\end{array}\right)^{2}\right) z^{n}
$$

is a $p$-Mahler function.

Regarding (1.1), (1.2) or (1.3), it is tempting to ask about the significance of the integer parameter $k$. Already in 1976, van der Poorten [37] suggested that two solutions of Mahler-type functional equations associated with essentially distinct parameters should be completely different. For instance, one may naturally expect [37] (and it is now proved [33]) that the two functions

$$
\sum_{n=0}^{\infty} z^{2^{n}} \text { and } \sum_{n=0}^{\infty} z^{3^{n}}
$$


are algebraically independent over $\mathbb{C}(z)$. This idea was later formalized by Loxton and van der Poorten who made a general conjecture whose one-dimensional version can be stated as follows.

Conjecture 1.1 (Loxton and van der Poorten). - Let $k$ and $l$ be two multiplicatively independent positive integers and $L$ be a number field. Let $F(z) \in L[[z]]$ be a locally analytic function that is both $k$-and $\ell$-Mahler. Then $F(z)$ must be a rational function.

We recall that two integers $k$ and $l$ larger than 1 are multiplicatively independent if there is no pair of positive integers $(n, m)$ such that $k^{n}=\ell^{m}$, or equivalently, if $\log (k) / \log (\ell) \notin \mathbb{Q}$. Conjecture 1.1 first appeared in print in 1987 in a paper of van der Poorten [38]. Since then it was explicitly studied in a number of different contexts including in some papers of Loxton [23], Becker [7], Randé [39], Bell [8] and the monograph of Everest et al. [16]. Independently, Zannier also considered a similar question in [43].

In this paper, our aim is to prove the following result.

Theorem 1.1. - Let $K$ be a field of characteristic zero and let $k$ and $l$ be two multiplicatively independent positive integers. Then a power series $F(z) \in$ $K[[z]]$ is both $k$-and $\ell$-Mahler if and only if it is a rational function.

Let us make few comments on this result.

- Taking $K$ to be a number field in Theorem 1.1 gives Conjecture 1.1.

- If $k$ and $\ell$ denote two multiplicatively dependent natural numbers, then a power series is $k$-Mahler if and only if it is also $\ell$-Mahler.

- As explained in more details in Section 2, one motivation for proving Theorem 1.1 is that it provides a far-reaching generalization of one fundamental result in the theory of sets of integers recognizable by finite automata: Cobham's theorem. Loxton and van der Poorten $[\mathbf{2 3}, \mathbf{3 8}]$ actually guessed that Conjecture 1.1 should be a consequence of some algebraic independence results for Mahler functions of several variables. In particular, they hoped to obtain a totally new proof of Cobham's theorem by using Mahler's method. Note, however, that our proof of Theorem 1.1 follows a totally different way and ultimately relies on Cobham's theorem, so we do not obtain an independent derivation of that result.

- Another important motivation for establishing Theorem 1.1 comes from the fact that these kind of statements, though highly natural and somewhat ubiquitous, are usually very difficult to prove. In particular, similar independence phenomena, involving two multiplicatively independent integers, are expected in various contexts but only very few results have 
been obtained up to now. As an illustration, we quote below three interesting open problems that rest on such a principle, all of them being widely open ${ }^{(*)}$. A long-standing question in dynamical systems is the socalled $\times 2 \times 3$ problem addressed by Furstenberg [17]: prove that the only Borel measures on $[0,1]$ that are simultaneously ergodic for $T_{2}(x)=2 x$ $(\bmod 1)$ and $T_{3}(x)=3 x(\bmod 1)$ are the Lebesgue measure and measures supported by those orbits that are periodic for both actions $T_{2}$ and $T_{3}$. The following problem, sometimes attributed to Mahler, was suggested by Mendès France in [30] (see also [2]): given a binary sequence $\left(a_{n}\right)_{n \geq 0} \in\{0,1\}^{\mathbb{N}}$, prove that

$$
\sum_{n=0}^{\infty} \frac{a_{n}}{2^{n}} \text { and } \sum_{n=0}^{\infty} \frac{a_{n}}{3^{n}}
$$

are both algebraic numbers only if both are rational numbers. The third problem we mention appeared implicitly in work of Ramanujan (see [41]): prove that both $2^{x}$ and $3^{x}$ are integers only if $x$ is a natural number. This is a particular instance of the four exponentials conjecture, a famous open problem in transcendence theory [40, Chapter 1, p. 15].

The outline of the paper is as follows. In Section 2, we briefly discuss the connection between Theorem 1.1 and Cobham's theorem. In Section 3, we describe our strategy for proving Theorem 1.1. Then the remaining Sections 4-11 are devoted to the different steps of the proof of Theorem 1.1.

\section{Connection with finite automata and Cobham's theorem}

One motivation for proving Theorem 1.1 is that it provides a far-reaching generalization of one fundamental result in the theory of sets of integers recognizable by finite automata. The aim of this section is to briefly describe this connection. For more details on automatic sets and automatic sequences, we refer the reader to the book of Allouche and Shallit [4].

Let $k \geq 2$ be a natural number. A set $\mathcal{N} \subset \mathbb{N}$ is said to be $k$-automatic if there is a finite-state machine that accepts as input the expansion of $n$ in base $k$ and outputs 1 if $n \in \mathcal{N}$ and 0 otherwise. For example, the set of Thue-Morse integers $1,2,4,7,8,11,13, \ldots$, formed by the integers whose sum of binary digits is odd, is 2-automatic. The associated automaton is given in Figure 1 below. It has two states. This automaton successively reads the binary digits of $n$ (starting, say, from the most significant digit and the initial

*. In all of these problems, the integers 2 and 3 may of course be replaced by any two multiplicatively independent integers larger than 1 . This list of problems is clearly not exhaustive and could be easily enlarged. 
state $\left.q_{0}\right)$ and thus ends the reading either in state $q_{0}$ or in state $q_{1}$. The initial state $q_{0}$ gives the output 0 , while $q_{1}$ gives the output 1 .

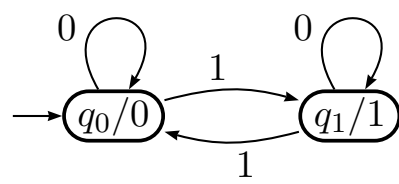

Figure 1. The finite-state automaton recognizing the set of ThueMorse integers.

Another typical 2-automatic set of integers is given by the powers of 2 : $1,2,4,8,16, \ldots$. Though these integers have very simple expansions in base 2 , one can observe that this is not the case when writing them in base 3 . One of the most important results in the theory of automatic sets formalizes this idea. It says that only very well-behaved sets of integers can be automatic with respect to two multiplicatively independent numbers. Indeed, in 1969 Cobham $[\mathbf{1 0}]$ proved the following result.

Theorem 2.1 (Cobham). - Let $k$ and $\ell$ be two multiplicatively independent integers. Then a set $\mathcal{N} \subseteq \mathbb{N}$ is both $k$-and $\ell$-automatic if and only if it is the union of a finite set and a finite number of arithmetic progressions.

The proof given by Cobham of his theorem is elementary but notoriously difficult and it remains a challenging problem to find a more natural/conceptual proof (see for instance the comment in Eilenberg [14, p. 118]). There are many interesting generalizations of this result. A very recent one is due to Durand [13] and we refer the reader to the introduction of $[\mathbf{1 3}]$ for a brief but complete discussion about such generalizations.

To end this section, let us briefly explain why Cobham's Theorem is a consequence of Theorem 1.1. Let us assume that $\mathcal{N} \subseteq \mathbb{N}$ is both $k$ - and $\ell$-automatic for multiplicatively independent natural numbers $k$ and $\ell$. Set $F(x):=\sum_{n \in \mathcal{N}} x^{n} \in \mathbb{Z}[[x]]$. Then it is known that $F(x)$ is both $k$ - and $\ell$ Mahler (see for instance [16, p. 232]). By Theorem 1.1, it follows that $F(x)$ is a rational function and thus the sequence of coefficients of $F(x)$ does satisfy a linear recurrence. Since the coefficients of $F(x)$ take only a two distinct values ( 0 and 1 ), we see that this linear recurrence is ultimately periodic. This exactly means that $\mathcal{N}$ is the union of a finite set and a finite number of arithmetic progressions, as claimed by Cobham's theorem.

\section{Sketch of proof of Theorem 1.1}

In this section, we describe the main steps of the proof of Theorem 1.1. 
Let $R$ be a ring and $\mathfrak{P}$ be an ideal of $R$. If $F(x)=\sum_{n=0}^{\infty} f(n) x^{n} \in R[[x]]$, then we denote by $F_{\mathfrak{P}}(x)$ the reduction of $F(x)$ modulo $\mathfrak{P}$, that is

$$
F_{\mathfrak{P}}(x)=\sum_{n=0}^{\infty}(f(n) \bmod \mathfrak{P}) x^{n} \in(R / \mathfrak{P})[[x]] .
$$

Let $K$ be a field of characteristic zero and $F(x) \in K[[x]]$ be both $k$ - and $\ell$-Mahler.

Step 0. This is a preliminary step. In the introduction, we defined Mahler functions as those satisfying Equation (1.3) but it is not always convenient to work with this general form of equations. In Sections 4 and 6 we show that there is no loss of generality to work with some more restricted types of functional equations. Also in Section 8, we prove that one can assume without loss of generality some additional assumptions on $k$ and $\ell$; namely that there are primes $p$ and $q$ such that $p$ divides $k$ but does not divide $\ell$ and $q$ divides $\ell$ but does not divide $k$.

Step 1. A first observation, proved in Section 5, is that the coefficients of the formal power series $F(x)$ only belong to some finitely generated $\mathbb{Z}$-algebra $R \subseteq K$. Then we prove the following useful local-global principle: $F(x)$ is a rational function if it has rational reduction modulo a sufficiently large set of maximal ideals of $R$. Using classical results of commutative algebra about Jacobson rings, we derive from our local-global principal that there is no loss of generality to assume that $K$ is a number field and that $R$ is the principal localization of a number ring.

Comment. Our strategy consists now in applying again our local-global principle. Indeed, since $R$ is the principal localization of a number ring, we have that the quotient ring $R / \mathfrak{P}$ is a finite field for every prime ideal $\mathfrak{P}$ of $R$. Our plan is thus to take advantage of the fact that $F_{\mathfrak{P}}(x)$ has coefficients in the finite set $R / \mathfrak{P}$ to prove that $F_{\mathfrak{P}}(x)$ is both a $k$ - and an $\ell$-automatic power series, for some prime ideals $\mathfrak{P}$. If this is the case, then Cobham's theorem applies and we get that $F_{\mathfrak{P}}(x)$ is a rational function. The localglobal principle actually implies that it is enough to prove that $F_{\mathfrak{P}}(x)$ is both $k$ - and $\ell$-automatic for infinitely many prime ideals $\mathfrak{P}$ of $R$.

Step 2. In Section 7, we underline the relation between $k$-Mahler, $k$-regular, and $k$-automatic power series. In particular, we show that every $k$-Mahler power series can be decomposed as

$$
F(x)=G(x) \cdot \Pi(x),
$$

where $G(x) \in R[[x]]$ is a $k$-regular power series and $\Pi(x) \in R[[x]]$ is the inverse of an infinite product of polynomials. Since $F(x)$ is also $\ell$-Mahler, we also have 
a similar decomposition

$$
F(x)=H(x) \cdot \Pi^{\prime}(x),
$$

where $H(x) \in R[[x]]$ is a $\ell$-regular power series and $\Pi^{\prime}(x) \in R[[x]]$ is the inverse of an infinite product of polynomials. Furthermore, the theory of regular power series implies that $G_{\mathfrak{P}}(x)$ is $k$-automatic and that $H_{\mathfrak{P}}(x)$ is $\ell$-automatic for every prime ideal $\mathfrak{P}$ of $R$.

In Section 11 we will split both infinite products $\Pi(x)$ and $\Pi^{\prime}(x)$ and get an expression of the form

$$
F(x)=G(x) \cdot \Pi_{1}(x) \cdot \Pi_{2}(x)=H(x) \cdot \Pi_{1}^{\prime}(x) \cdot \Pi_{2}^{\prime}(x)
$$

where $\Pi_{1}(x), \Pi_{2}(x), \Pi_{1}^{\prime}(x), \Pi_{2}^{\prime}(x) \in R[[x]]$ are inverses of some other infinite products of polynomials.

Step 3. In Section 9, we look at the singularities of Mahler functions at roots of unity. We use asymptotic techniques to show that one can reduce to the case of considering Mahler equations whose singularities at roots of unity have a restricted form. This ensures, using some results of Section 7 , that $\Pi_{1}(x)$ is $k$-automatic and that $\Pi_{1}^{\prime}(x)$ is $\ell$-automatic when reduced modulo every prime ideal $\mathfrak{P}$ of $R$.

Step 4. In our last step, we use Chebotarev's density theorem in order to ensure the existence of an infinite set $\mathcal{S}$ of prime ideals of $R$ such that $\Pi_{2}(x)$ is $k$-automatic and $\Pi_{2}^{\prime}(x)$ is $\ell$-automatic when reduced modulo every ideal $\mathfrak{P} \in \mathcal{S}$.

Conclusion. Since the product of $k$-automatic power series is $k$-automatic, we infer from Steps 2, 3 and 4 that for every prime ideals $\mathfrak{P} \in \mathcal{S}$ the power series $F_{\mathfrak{P}}(x)$ is both $k$ - and $\ell$-automatic. By Cobham's theorem, $F_{\mathfrak{P}}(x)$ is rational for every such prime ideal. Then the local-global principle ensures that $F(x)$ is rational, as desired.

\section{Preliminary reduction for the form of Mahler equations}

In the introduction, we define $k$-Mahler functions as power series satisfying a functional equation of the form given in (1.3). In the literature, they are sometimes defined as solutions of a more restricted type of functional equations. We recall here that these apparently stronger conditions on the functional equations actually lead to the same class of functions. In the sequel, it will thus be possible to work without loss of generality with these more restricted type of equations. 
Lemma 4.1. - Let us assume that $F(x)$ satisfies a $k$-Mahler equation as in (1.3). Then there exist polynomials $P_{0}(x), \ldots, P_{n}(x)$ in $K[x]$, with $\operatorname{gcd}\left(P_{0}(x), \ldots, P_{n}(x)\right)=1$ and $P_{0}(x) P_{n}(x) \neq 0$, and such that

$$
\sum_{i=0}^{n} P_{i}(x) F\left(x^{k^{i}}\right)=0 .
$$

Proof. - Let us assume that $F(x)$ satisfies a $k$-Mahler equation as in (1.3). There thus exist some nonnegative integer $n$ and polynomials $A(x), A_{0}(x), \ldots, A_{n}(x)$ in $K[x]$, with $A_{n}(x)$ nonzero, such that

$$
\sum_{i=0}^{n} A_{i}(x) F\left(x^{k^{i}}\right)=A(x) .
$$

We first show that we can assume that $A(x)=0$. Indeed, let us assume that $A(x) \neq 0$. Applying the operator $x \mapsto x^{k}$ to this equation, we get that

$$
\sum_{i=0}^{n} A_{i}\left(x^{k}\right) F\left(x^{k^{i+1}}\right)=A\left(x^{k}\right) .
$$

Multiplying the first equation by $A\left(x^{k}\right)$ and the second by $A(x)$ and subtracting, we obtain the new equation

$$
\sum_{i=0}^{n+1} B_{i}(x) F\left(x^{k^{i}}\right)=0,
$$

where $B_{i}(x):=A_{i}(x) A\left(x^{k}\right)-A_{i}\left(x^{x}\right) A(x)$ for every integer $i, 1 \leq i \leq n$ and where $B_{n+1}:=A_{n}\left(x^{k}\right) A(x) \neq 0$. We can thus assume without loss of generality that $A(x)=0$.

Now among all such nontrivial relations of the form

$$
\sum_{i=0}^{n} P_{i}(x) F\left(x^{k^{i}}\right)=0,
$$

we choose one with $n$ minimal. Thus $P_{n}(x)$ is nonzero. We claim $P_{0}(x)$ is nonzero. Let us assume this is not the case. Pick the smallest integer $j$ such that $P_{j}(x)$ is nonzero. By assumption, $j>0$. Then there is some nonnegative integer $a$ such that the coefficient of $x^{a}$ in $P_{j}(x)$ is nonzero. Let $b$ be the unique integer such that $a \equiv b \bmod k$ and $0 \leq b<k$. Let us define the operator $\Lambda_{b}$ from $K[[x]]$ into itself by

$$
\Lambda_{b}\left(\sum_{i=0}^{\infty} f(i) x^{i}\right):=\sum_{i=0}^{\infty} f(k i+b) x^{i} .
$$


Then every $F(x) \in K[[x]]$ has a unique decomposition as

$$
F(x)=\sum_{b=0}^{k-1} x^{b} \Lambda_{b}(F)\left(x^{k}\right),
$$

which implies that

$$
\Lambda_{b}\left(F(x) G\left(x^{k}\right)\right)=\Lambda_{b}(F(x)) G(x)
$$

for every pair of power series $F(x), G(x) \in K[[x]]$. Applying $\Lambda_{b}$ to Equation (4.6), we thus get that

$$
0=\Lambda_{b}\left(\sum_{i=j}^{n} P_{i}(x) F\left(x^{k^{i}}\right)\right)=\sum_{i=j-1}^{n-1} \Lambda_{b}\left(P_{i+1}(x)\right) F\left(x^{k^{i}}\right) .
$$

By construction, $\Lambda_{b}\left(P_{j}(x)\right)$ is nonzero, which shows that this relation is nontrivial. This contradicts the minimality of $n$. It follows that $P_{0}(x)$ is nonzero.

Furthermore, if $\operatorname{gcd}\left(P_{0}(x), \ldots, P_{n}(x)\right)=D(x) \neq 0$, it suffices to divide (4.6) by $D(x)$ to obtain an equation with the desired properties. This ends the proof.

\section{Reduction to the number field case}

In this section, we show that we may restrict our attention to the case where the base field $K$ is replaced by a number field and more precisely by a principal localization of a number ring.

Theorem 5.1. - Let us assume that the conclusion of Theorem 1.1 holds whenever the field $K$ is replaced by a principal localization of a number ring. Then Theorem 1.1 is true.

We first observe that the coefficients of a Mahler function in $K[[x]]$ actually belong to some finitely generated $\mathbb{Z}$-algebra $R \subseteq K$.

Lemma 5.1. - Let $K$ be a field of characteristic zero, let $k \geq 2$ be an integer, and let $F(x) \in K[[x]]$ be a $k$-Mahler power series. Then there exists a finitely generated $\mathbb{Z}$-algebra $R \subseteq K$ such that $F(x) \in R[[x]]$.

Proof. - We first infer from Lemma 4.1 that there exist a natural number $n$ and polynomials $P_{0}(x), \ldots, P_{n}(x) \in K[x]$ with $P_{0}(x) P_{n}(x) \neq 0$ such that

$$
\sum_{i=0}^{n} P_{i}(x) F\left(x^{k^{i}}\right)=0 \text {. }
$$


Let $d$ be a natural number that is strictly greater than the degrees of the polynomials $P_{0}(x), \ldots, P_{n}(x)$. Let $R$ denote the smallest $\mathbb{Z}$-algebra countaining:

- the coefficients of $P_{0}(x), \ldots, P_{n}(x)$;

- the coefficients $f(0), \ldots, f(d)$;

- the multiplicative inverses of all nonzero coefficients of $P_{0}(x)$.

By definition, $R \subseteq K$ is a finitely generated $\mathbb{Z}$-algebra. We claim that $F(x) \in R[[x]]$. To see this, suppose that this is not the case. Then there is some smallest natural number $n_{0}$ such that $f\left(n_{0}\right) \notin R$. Furthermore, $n_{0}>d$. Consider the equation

$$
P_{0}(x) F(x)=-\sum_{i=1}^{n} P_{i}(x) F\left(x^{k^{i}}\right) .
$$

Let $i$ denote the order of $P_{0}(x)$ at $x=0$ and let $c \neq 0$ denote the coefficient of $x^{i}$ in $P_{0}(x)$. Then if we extract the coefficient of $x^{n_{0}+i}$ in Equation (5.7), we see that $c f\left(n_{0}\right)$ can be expressed as an $R$-linear combination of $f(0), \ldots, f\left(n_{0}-1\right)$. Hence $c f\left(n_{0}\right)$ belongs to $R$ by the minimality of $n_{0}$. Since $c^{-1} \in R$ we see that $f\left(n_{0}\right) \in R$, a contradiction. This ends the proof.

We now prove that the height of a rational function which satisfies a Mahlertype equation can be bounded by the maximal of the degrees of the polynomials defining the underlying equation.

Lemma 5.2. - Let $K$ be a field, let $n$ and $d$ be natural numbers, and let $P_{0}(x), \ldots, P_{n}(x)$ be polynomials in $K[x]$ of degree at most $d$ with $P_{0}(x) P_{n}(x) \neq$ 0 . Suppose that $F(x) \in K[[x]]$ satisfies the Mahler-type equation

$$
\sum_{i=0}^{n} P_{i}(x) F\left(x^{k^{i}}\right)=0 .
$$

If $F(x)$ is rational, then there exist polynomials $A(x)$ and $B(x)$ of degree at most $d$ with $B(0)=1$ such that $F(x)$ is the power series expansion of $A(x) / B(x)$.

Proof. - Without any loss of generality we can assume that $F(x)$ is not identically zero. If $F(x)$ is rational, then there exist two polynomials $A(x)$ and $B(x)$ in $K[x]$ with ged 1 and with $B(0)=1$ such that $F(x)=A(x) / B(x)$. Observe that

$$
\sum_{i=0}^{n} P_{i}(x) A\left(x^{k^{i}}\right) / B\left(x^{k^{i}}\right)=0 .
$$


Multiplying both sides of this equation by the product $B(x) B\left(x^{k}\right) \cdots B\left(x^{k^{n}}\right)$, we see that $B\left(x^{k^{n}}\right)$ divides

$$
P_{n}(x) A\left(x^{k^{n}}\right) B(x) \cdots B\left(x^{k^{n-1}}\right) .
$$

Since $\operatorname{gcd}(A(x), B(x))=1$ and $A(x)$ is nonzero, we actually have that $B\left(x^{k^{n}}\right)$ divides

$$
P_{n}(x) B(x) \cdots B\left(x^{k^{n-1}}\right) .
$$

Let $d_{0}$ denote the degree of $B(x)$. Then we have

$$
\begin{aligned}
k^{n} d_{0} & \leq \operatorname{deg}\left(P_{n}(x)\right)+\sum_{i=0}^{n-1} \operatorname{deg}\left(B\left(x^{k^{i}}\right)\right) \\
& \leq d+d_{0}\left(1+k+\cdots+k^{n-1}\right) \\
& =d+d_{0}\left(k^{n}-1\right) /(k-1) .
\end{aligned}
$$

Thus

$$
d_{0}\left(k^{n+1}-2 k^{n}+1\right) /(k-1) \leq d,
$$

which implies $d_{0} \leq d$ since $\left(k^{n+1}-2 k^{n}+1\right) /(k-1) \geq 1$ for every integer $k \geq 2$. A symmetric argument gives the same upper bound for the degree of $A(x)$.

We derive from Lemma 5.2 a useful local-global principle for the rationality of Mahler functions with coefficients in a finitely generated $\mathbb{Z}$-algebra.

Lemma 5.3. - Let $K$ be a field, let $k \geq 2$ be an integer, and let $R \subseteq K$ be a finitely generated $\mathbb{Z}$-algebra. Let us assume that $F(x) \in R[[x]]$ has the following properties.

(i) There exist a natural number $d$ and polynomials $P_{0}(x), \ldots, P_{n}(x) \in R[x]$ with $P_{0}(x) P_{n}(x) \neq 0$ such that

$$
\sum_{i=0}^{n} P_{i}(x) F\left(x^{k^{i}}\right)=0 .
$$

(ii) There exists an infinite set $\mathcal{S}$ of maximal ideals of $R$ such that $F(x) \bmod I$ is a rational power series in $(R / I)[[x]]$ for every $I \in \mathcal{S}$.

(iii) One has $\bigcap_{I \in \mathcal{S}} I=\{0\}$.

Then $F(x)$ is a rational function. 
Proof. - Let $d$ be a natural number that is strictly greater than the degrees of all polynomials $P_{0}(x), \ldots, P_{n}(x)$. By (ii), we have that for each maximal ideal $I$ in $\mathcal{S}, F(x) \bmod I$ is a rational function. Thus by (i) and Lemma 5.2, we see that for each maximal ideal $I$ in $\mathcal{S}$, there exist two polynomials $A_{I}(x)$ and $B_{I}(x) \in(R / I)[x]$ of degree at most $d$ with $B_{I}(0)=1$ and such that $F(x) \equiv A_{I}(x) / B_{I}(x) \bmod I$. In particular, if $F(x)=\sum_{j \geq 0} f(j) x^{j}$, we see that the sequences in the set $\left\{(f(d+1+i+j) \bmod I)_{j \geq 0} \mid i=0, \ldots, d\right\}$ are linearly dependent over $R / I$. Thus the determinant of each $(d+1) \times(d+1)$ submatrix of the infinite matrix

$$
M:=\left(\begin{array}{cccc}
f(d+1) & f(d+2) & f(d+3) & \cdots \\
f(d+2) & f(d+3) & f(d+4) & \cdots \\
\vdots & \vdots & \vdots & \cdots \\
f(2 d+1) & f(2 d+2) & f(2 d+3) & \cdots
\end{array}\right)
$$

lies in the maximal ideal $I$. Since this holds for every maximal ideal $I$ in $\mathcal{S}$, we infer from (iii) that every $(d+1) \times(d+1)$ minor of $M$ vanishes. It follows that $M$ has rank at most $d$ and thus the rows of $M$ are linearly dependent over the field of fractions of $R$. In particular, there exist $c_{0}, \ldots, c_{d} \in R$, not all zero, such that

$$
\sum_{i=0}^{d} c_{i} f(d+1+i+j)=0
$$

for all $j \geq 0$. Letting $B(x):=c_{d}+c_{d-1} x+\cdots+c_{0} x^{d}$, we see that $B(x) F(x)$ is a polynomial. Hence $F(x)$ is a rational function. This ends the proof.

We are now ready to prove the main result of this section.

Proof of Theorem 5.1. - Let $K$ be a field of characteristic zero and let $F(x) \in K[[x]]$ be a power series that is both $k$ - and $\ell$-Mahler for some multiplicatively independent natural numbers $k$ and $\ell$. By Lemma 4.1, there are natural numbers $n$ and $m$ and polynomials $P_{0}(x), \ldots, P_{n}(x)$ and $Q_{0}(x), \ldots, Q_{m}(x)$ with $P_{0}(x) P_{n}(x) Q_{0}(x) Q_{m}(x) \neq 0$ and such that

$$
\sum_{i=0}^{n} P_{i}(x) F\left(x^{k^{i}}\right)=\sum_{j=0}^{m} Q_{j}(x) F\left(x^{\ell^{j}}\right)=0 .
$$

Then by Lemma 5.1 , there is a finitely generated $\mathbb{Z}$-algebra $R \subseteq K$ such that $F(x) \in R[[x]]$. By adding all the coefficients of $P_{0}(x), \ldots, P_{n}(x)$ and of $Q_{0}(x), \ldots, Q_{m}(x)$ to $R$, we can assume that $P_{i}(x)$ and $Q_{j}(x)$ are in $R[x]$ for $(i, j) \in\{1, \ldots, n\} \times\{1, \ldots, m\}$. By inverting the nonzero integers in $R$, we can assume that $R$ is a finitely generated $\mathbb{Q}$-algebra.

Let $\mathcal{M} \subseteq \operatorname{Spec}(R)$ denote the collection of maximal ideals of $R$. Since $R$ is a finitely generated $\mathbb{Q}$-algebra, $R$ is a Jacobson ring and $R / I$ is a finite extension 
of $\mathbb{Q}$ for every $I \in \mathcal{M}$ (see [15, Theorem 4.19, p. 132]). Thus, for each maximal ideal $I$ of $R$, the quotient field $R / I$ is a number field. If we assume that the conclusion of Theorem 1.1 holds when the base field is a number field, then we get that $F(x) \bmod I$ is a rational function in $(R / I)[[x]]$ for it is clearly both $k$ - and $\ell$-Mahler ${ }^{*}$. Since $R$ is a Jacobson ring that is also a domain, we have that $\bigcap_{I \in \mathcal{M}} I=\{0\}$ (c.f. [15, p. 132]). Then Lemma 5.3 implies that $F(x)$ is a rational function in $R[[x]]$. This shows it is sufficient to prove Theorem 1.1 in the case that $K$ is a number field.

We can thus assume that $F(x) \in K[[x]]$ where $K$ is a number field. Now, if we apply again Lemma 5.1, we see that there is a finitely generated $\mathbb{Z}$ algebra $R \subseteq K$ such that $F(x) \in R[[x]]$. Furthermore, every finitely generated $\mathbb{Z}$-subalgebra of a number field $K$ has a generating set of the form $\left\{a_{1} / b, \ldots, a_{t} / b\right\}$, where $b$ is a nonzero (rational) integer and $a_{1}, \ldots, a_{t}$ are algebraic integers in $K$. Thus $R$ is a subalgebra of a principal localization of a number ring, that is $R \subseteq\left(\mathcal{O}_{K}\right)_{b}$, where $\mathcal{O}_{K}$ denotes the ring of algebraic integers in $K$. Thus to establish Theorem 1.1 it is sufficient to prove the following result: let $k$ and $\ell$ be two multiplicatively independent natural numbers, let $R$ be a principal localization of a number ring, and let $F(x) \in R[[x]]$, then if $F(x)$ is both $k$ - and $\ell$-Mahler it is a rational function. This concludes the proof.

\section{Further reductions for the form of Mahler equations}

In this section, we refine the results of Section 4. We show that a power series satisfying a Mahler equation of the form given in (4.5) is also solution of a more restricted type of functional equations.

Lemma 6.1. - Let $K$ be a field and $k \geq 2$ be an integer. Let us assume that $F(x):=\sum_{i \geq 0} f(i) x^{i} \in K[[x]]$ satisifes a $k$-Mahler equation of the form

$$
\sum_{i=0}^{n} P_{i}(x) F\left(x^{k^{i}}\right)=0,
$$

where $P_{0}(x), \ldots, P_{n}(x) \in K[x], \operatorname{gcd}\left(P_{0}(x), \ldots, P_{n}(x)\right)=1$ and $P_{0}(x) P_{n}(x) \neq$ 0 . Then there exists a natural number $N$ such that, for every integer $a>N$ with $f(a) \neq 0, F(x)$ can be decomposed as

$$
F(x)=T_{a}(x)+x^{a} F_{0}(x),
$$

\footnotetext{
*. Note that since $P_{0}(0) Q_{0}(0) \neq 0$, we may assume that $P_{0}(0)=Q_{0}(0)=1$ by multiplying the left-hand side of $(5.8)$ by $1 / P_{0}(0)$ and the right-hand side of $(5.8)$ by $1 / Q_{0}(0)$. This ensures that, for each functional equation, not all the coefficients vanish when reduced modulo a maximal ideal $I$ of $R$. Hence $F(x) \bmod I$ is both $k$ - and $\ell$-Mahler.
} 
where $T_{a}(x) \in K[x]$ and $F_{0}(x)$ has nonzero constant term and satisfies a $k$ Mahler equation

$$
\sum_{i=0}^{m} Q_{i}(x) F_{0}\left(x^{k^{i}}\right)=0
$$

for some natural number $m$ and polynomials $Q_{0}, \ldots, Q_{m} \in K[x]$ satisfying the following conditions.

(i) One has $Q_{0}(0)=1$.

(ii) If $\alpha \neq 0$ and $P_{0}(\alpha)=0$, then $Q_{0}(\alpha)=0$.

(iii) If $\alpha \neq 0, P_{0}(\alpha)=0$ and $\alpha^{k}=\alpha$, then $Q_{j}(\alpha) \neq 0$ for some $j \in$ $\{1, \ldots, m\}$.

Proof. - By assumption, we have that $F(x)$ satisfies a $k$-Mahler equation

$$
\sum_{i=0}^{n} P_{i}(x) F\left(x^{k^{i}}\right)=0,
$$

where $P_{0}(x) P_{n}(x)$ is nonzero. Let $N$ denote the order of vanishing of $P_{0}(x)$ at $x=0$. Suppose that $a \geq N$ and $f(a) \neq 0$. Then we have that

$$
F(x)=T_{a}(x)+x^{a} F_{0}(x),
$$

for some polynomial $T_{a}(x)$ of degree $a-1$ and some power series $F_{0}(x)$ with nonzero constant term. Then we have

$$
\sum_{i=0}^{n} P_{i}(x)\left(T_{a}\left(x^{k^{i}}\right)+x^{k^{i} \cdot a} F_{0}\left(x^{k^{i}}\right)\right)=0,
$$

which we can write as

$$
\sum_{i=0}^{n} P_{i}(x) x^{k^{i} \cdot a} F_{0}\left(x^{k^{i}}\right)=C(x),
$$

where $C(x)$ denotes the polynomial

$$
C(x):=-\sum_{i=0}^{n} P_{i}(x) T_{a}\left(x^{k^{i}}\right) .
$$

Set $S(x):=P_{0}(x) x^{-N}$. By definition of $N, S(x)$ is a polynomial with $S(0) \neq 0$. Then if we divide both sides of Equation (6.9) by $x^{a+N}$, we obtain that

$$
S(x) F_{0}(x)+\sum_{i=1}^{n} P_{i}(x) x^{k^{i} a-a-N} F_{0}\left(x^{k^{i}}\right)=x^{-a-N} C(x) .
$$


Observe that the left-hand side is a power series with constant term $S(0) F_{0}(0) \neq 0$ and thus $C_{0}(x):=x^{-a-N} C(x)$ is a polynomial with $C_{0}(0) \neq 0$. Applying the operator $x \mapsto x^{k}$, we also obtain that

$$
S\left(x^{k}\right) F_{0}\left(x^{k}\right)+\sum_{i=1}^{n} P_{i}\left(x^{k}\right) x^{k^{i+1} a-k a-k N} F_{0}\left(x^{k^{i+1}}\right)=C_{0}\left(x^{k}\right) .
$$

Multiplying $(6.10)$ by $C_{0}\left(x^{k}\right)$ and $(6.11)$ by $C_{0}(x)$ and then subtracting, we get that

$$
\begin{aligned}
& C_{0}\left(x^{k}\right) S(x) F_{0}(x)+\sum_{i=1}^{n} C_{0}\left(x^{k}\right) P_{i}(x) x^{k^{i} a-a-N} F_{0}\left(x^{k^{i}}\right) \\
& -C_{0}(x) S\left(x^{k}\right) F_{0}\left(x^{k}\right)-\sum_{i=1}^{n} C_{0}(x) P_{i}\left(x^{k}\right) x^{k^{i+1} a-k a-k N} F_{0}\left(x^{k^{i+1}}\right)=0 .
\end{aligned}
$$

Since $C_{0}(0)$ and $S(0)$ are nonzero, we see that $F_{0}(x)$ satisfies a non-trivial $k$-Mahler equation

$$
\sum_{i=0}^{n+1} Q_{i}(x) F_{0}\left(x^{k^{i}}\right)=0
$$

where

$$
Q_{0}(x):=\frac{C_{0}\left(x^{k}\right) S(x)}{\operatorname{gcd}\left(C_{0}(x), C_{0}\left(x^{k}\right)\right)}
$$

and

$$
Q_{1}(x):=\frac{C_{0}\left(x^{k}\right) P_{1}(x) x^{k^{i} a-a-N}-C_{0}(x) S\left(x^{k}\right)}{\operatorname{gcd}\left(C_{0}(x), C_{0}\left(x^{k}\right)\right)},
$$

and, for $i \in\{2, \ldots, n+1\}$,

$$
Q_{i}(x):=\frac{x^{k^{i} a-k a-N}\left(C_{0}\left(x^{k}\right) x^{(k-1) a} P_{i}(x)-C_{0}(x) P_{i-1}\left(x^{k}\right)\right)}{\operatorname{gcd}\left(C_{0}(x), C_{0}\left(x^{k}\right)\right)},
$$

with the convention that $P_{n+1}(x):=0$. By construction, $Q_{0}(0) \neq 0$, which we may assume to be equal to 1 by multiplying our equation by $1 / Q_{0}(0)$. Since $S(x)$ divides $Q_{0}(x)$, we have that if $P_{0}(\alpha)=0$ for some nonzero $\alpha$ then $Q_{0}(\alpha)=0$. Finally, suppose that $P_{0}(\alpha)=0$ for some nonzero $\alpha$ such that $\alpha^{k}=\alpha$. We claim that $Q_{j}(\alpha)$ is nonzero for some $j \in\{1, \ldots, n+1\}$. Note that since $\operatorname{gcd}\left(P_{0}(x), \ldots, P_{n}(x)\right)=1$, there is some smallest positive integer $i$ such that $P_{i}(\alpha)$ is nonzero. We claim that $Q_{i}(\alpha) \neq 0$. Indeed, otherwise $\alpha$ would be a root of $C_{0}(x) / \operatorname{gcd}\left(C_{0}(x), C_{0}\left(x^{k}\right)\right)$, but this is impossible since $\alpha^{k}=\alpha$. This ends the proof.

Corollary 6.1. - Let $K$ be a field and let $k$ and $\ell$ be multiplicatively independent natural numbers. Let $F(x) \in K[[x]]$ be a power series that is both $k$ - 
and $\ell$-Mahler and that is not a polynomial. Then there is a natural number a such that $F(x)$ can be decomposed as

$$
F(x)=T_{a}(x)+x^{a} F_{0}(x),
$$

where $T_{a}(x)$ is a polynomial of degree $a-1, F_{0}(x)$ satisfies a $k$-Mahler equation as in Lemma 6.1, and $F_{0}(x)$ also satisfies an $\ell$-Mahler equation of the form

$$
\sum_{i=0}^{r} R_{i}(x) F_{0}\left(x^{\ell^{i}}\right)=0
$$

with $R_{0}(x), \ldots, R_{r}(x) \in K[x]$ and $R_{0}(0)=1$.

Proof. - The result follows directly by applying Lemma 6.1 twice to $F(x)$, viewed respectively as a $k$-Mahler and an $\ell$-Mahler function, and then by choosing $a$ large enough.

\section{Links with automatic and regular power series}

The aim of this section is to underline the relation between $k$-Mahler, $k$ regular, and $k$-automatic power series. We gather some useful facts about automatic and regular power series that will turn out to be useful for proving Theorem 1.1. We also observe that every $k$-Mahler power series can be decomposed as the product of a $k$-regular power series of a special type and the inverse of an infinite product of polynomials. Such a decomposition will play a key role in the proof of Theorem 1.1.

7.1. Automatic and regular power series. - We recall here basic facts about regular power series, which were introduced by Allouche and Shallit [5] (see also [6] and [4, Chapter 16]). They form a distinguished class of $k$-Mahler power series as well as a natural generalization of $k$-automatic power series.

A useful way to characterize $k$-automatic sequences, due to Eilenberg [14], is given in terms of the so-called $k$-kernel.

Definition 7.1. - Let $k \geq 2$ be an integer and let $\mathbf{f}=(f(n))_{n \geq 0}$ be a sequence with values in a set $E$. The $k$-kernel of $\mathbf{f}$ is defined as the set

$$
\left\{\left(f\left(k^{a} n+b\right)\right)_{n \geq 0} \mid a \geq 0, b \in\{0, \ldots, a-1\}\right\} .
$$

Theorem 7.1 (Eilenberg). - A sequence is $k$-automatic if and only if its $k$-kernel is finite.

This characterization gives rise to the following natural generalization of automatic sequences introduced by Allouche and Shallit [5]. 
Definition 7.2. - Let $R$ be a commutative ring and let $\mathbf{f}=(f(n))_{n \geq 0}$ be a $R$-valued sequence. Then $\mathbf{f}$ is said to be $k$-regular if the dimension of the $R$-module spanned by its $k$-kernel is finite.

In the sequel, we will say that a power series $F(x) \in R[[x]]$ is $k$-regular (respectively $k$-automatic) if its sequence of coefficients is $k$-regular (respectively $k$-automatic). In the following proposition, we collect some useful general facts about $k$-regular power series.

Proposition 7.1. - Let $R$ be a commutative ring and $k \geq 2$ be an integer. Then the following properties hold.

(i) If $F(x) \in R[[x]]$ is $k$-regular and $I$ is an ideal of $R$, then $F(x) \bmod I \in$ $(R / I)[[x]]$ is k-regular.

(ii) If $F(x) \in R[[x]]$ is $k$-regular, then the coefficients of $F(x)$ take only finitely many distinct values if and only if $F(x)$ is $k$-automatic.

(iii) If $F(x)=\sum_{i \geq 0} f(i) x^{i}$ and $G(x)=\sum_{i \geq 0} g(i) x^{i}$ are two $k$-regular power series in $R[[x]]$, then the Cauchy product

$$
F(x) G(x):=\sum_{i=0}^{\infty}\left(\sum_{j=0}^{i}\left(\begin{array}{l}
i \\
j
\end{array}\right) f(j) g(i-j)\right) x^{i}
$$

is k-regular.

Proof. - The property (i) follows directly from the definition of a $k$-regular sequence, while (ii) and (iii) correspond respectively to Theorem 16.1.5 and Corollary 16.4.2 in [4].

In Section 9, we will need to use that $k$-regular sequences with complex values do have strict restrictions on the growth of their absolute values, a fact evidenced by the following result.

Proposition 7.2. - Let $k \geq 2$ be a natural number and let $F(x) \in \mathbb{C}[[x]]$ be a k-regular power series. Then $F(x)$ is analytic in the open unit disc and there exist two positive real numbers $C$ and $m$ such that

$$
|F(x)|<C(1-|x|)^{-m},
$$

for all $x \in B(0,1)$.

Proof. - Let $F(x)=\sum_{i=0}^{\infty} f(i) x^{i} \in \mathbb{C}[[x]]$ be a $k$-regular power series. Then there is some positive constant $A$ and some integer $d>0$ such that

$$
|f(i)| \leq A(i+1)^{d}
$$


for every nonnegative integer $i$ (see [4, Theorem 16.3.1]). This immediately gives that $F(x)$ is analytic in the open unit disc. Moreover, for $x \in B(0,1)$,

$$
|F(x)| \leq \sum_{i=0}^{\infty} A(i+1)^{d}|x|^{i} \leq \sum_{i=0}^{\infty} A d !\left(\begin{array}{c}
i+d \\
d
\end{array}\right)|x|^{i}=A d !(1-|x|)^{-d-1} .
$$

The result follows.

7.2. Becker power series. - Becker [7, Theorem 1] showed that a $k$ regular power series is necessarily $k$-Mahler. In addition to this, he proved [7, Theorem 2] the following partial converse. The general converse does not hold.

Theorem 7.2 (Becker). - Let $K$ be a field, let $k$ be a natural number $\geq 2$, and let $F(x) \in K[[x]]$ be a power series that satisfies a $k$-Mahler equation of the form

$$
F(x)=\sum_{i=1}^{n} P_{i}(x) F\left(x^{k^{i}}\right)
$$

for some polynomials $P_{1}(x), \ldots, P_{n}(x) \in K[x]$. Then $F(x)$ is a $k$-regular power series.

Definition 7.3. - In honour of Becker's result, a power series $F(x) \in K[[x]]$ that satisfies an equation of the form given in Equation (7.12) will be called a $k$-Becker power series.

Theorem 7.2 shows that the set of $k$-Becker power series is contained in the set of $k$-regular power series. However, the converse is not true. As an example, we provide the following result that will also be used in Section 11.

Proposition 7.3. - Let $k$ be a natural number, and let $\omega \in \mathbb{C}$ be a root of unity with the property that if $j \geq 1$ then $\omega^{k^{j}} \neq \omega$. Then

$$
\left(\prod_{j=0}^{\infty}\left(1-\omega x^{k^{j}}\right)\right)^{-1}
$$

is $k$-regular but is not $k$-Becker.

Proof. - Since $\omega$ is a root of unity, the sequence $\omega, \omega^{k}, \omega^{k^{2}}, \ldots$ is eventually periodic and there is some smallest natural number $N$ such that

$$
\omega^{k^{2 N}}=\omega^{k^{N}} .
$$

Set $\beta:=\omega^{k^{N}}$ and let us consider the polynomial

$$
Q(x)=(1-\beta x)\left(1-\beta x^{k}\right) \cdots\left(1-\beta x^{k^{N-1}}\right) .
$$


Then

$$
\frac{Q\left(x^{k}\right)}{Q(x)}=\frac{1-\beta x^{k^{N}}}{1-\beta x}
$$

Since

$$
1-\beta x^{k^{N}}=1-(\beta x)^{k^{N}},
$$

we see that $Q\left(x^{k}\right) / Q(x)$ is a polynomial.

Since

$$
1-(\beta x)^{k^{N}}=\frac{Q\left(x^{k}\right)}{Q(x)} \cdot(1-\beta x),
$$

we get that $(1-\omega x)$ divides the polynomial $Q\left(x^{k}\right)(1-\beta x) / Q(x)$. Furthermore, $(1-\omega x)$ cannot divide $(1-\beta x)$ since by assumption $\omega \neq \beta$. By Euclid's lemma, we thus obtain that

$$
\frac{Q\left(x^{k}\right)}{Q(x)}=(1-\omega x) S(x)
$$

for some polynomial $S(x)$.

Set $G(x):=Q(x)^{-1} F(x)$. Since $F(x)$ satisfies the $k$-Mahler recurrence

$$
F\left(x^{k}\right)=(1-\omega x) F(x)
$$

we see that

$$
G\left(x^{k}\right)=Q\left(x^{k}\right)^{-1}(1-\omega x) Q(x) G(x)
$$

or equivalently,

$$
G(x)=S(x) G\left(x^{k}\right) .
$$

Thus $G(x)$ is a $k$-Becker power series. By Proposition $7.1, F(x)$ is $k$-regular as it is a product of a polynomial (which is $k$-regular) and a $k$-regular power series.

On the other hand, $F(x)$ cannot be a $k$-Becker power series. To see this, suppose that $F(x)$ satisfies an equation of the form

$$
F(x)=\sum_{i=1}^{d} P_{i}(x) F\left(x^{k^{i}}\right) .
$$

Now, dividing both sides by $F\left(x^{k}\right)$, the right-hand side becomes a polynomial in $x$, while the left-hand side is $(1-\omega x)^{-1}$, a contradiction. The result follows.

In Section 9, we will need the following basic result about $k$-Becker power series. 
Lemma 7.1. - Let $k \geq 2$ and let us assume that $F(x) \in K[[x]]$ satisfies a $k$-Mahler equation of the form

$$
F(x)=\sum_{i=1}^{n} a_{i} F\left(x^{k^{i}}\right)
$$

for some constants $a_{1}, \ldots, a_{n} \in K$. Then $F(x)$ is constant.

Proof. - Let us denote by $F(x)=\sum_{i>0} f(i) x^{i}$ the power series expansion of $F(x)$. If $F(x)$ were non-constant, there would be some smallest positive integer $i$ such that $f(i) \neq 0$. Thus $F(x)=\lambda+x^{i} F_{0}(x)$ for some $\lambda$ in $K$ and some $F_{0}(x) \in K[[x]]$. But taking the coefficient of $x^{i}$ in the right-hand side of the equation

$$
F(x)=\sum_{i=1}^{n} a_{i} F\left(x^{k^{i}}\right),
$$

we see that $f(i)=0$, a contradiction. The result follows.

Though there are some Mahler functions that are not Becker functions, the following result shows that every $k$-Mahler power series can be decomposed as the product of a $k$-Becker power series and the inverse of an infinite product of polynomials. This decomposition will turn out to be very useful to prove Theorem 1.1. We note that a similar result also appears as Theorem 31 in the Ph. D. Thesis of Dumas [11].

Proposition 7.4. - Let $k$ be a natural number, let $K$ be a field, and let $F(x) \in K[[x]]$ be a $k$-Mahler power series satisfying an equation of the form

$$
\sum_{i=0}^{n} P_{i}(x) F\left(x^{k^{i}}\right)=0,
$$

where $P_{0}(x), \ldots, P_{n}(x) \in K[x]$ and $P_{0}(0)=1$. Then there is a $k$-Becker power series $G(x)$ such that

$$
F(x)=\left(\prod_{i=0}^{\infty} P_{0}\left(x^{k^{i}}\right)\right)^{-1} G(x) .
$$

Proof. - Since $P_{0}(0)=1$, the infinite product

$$
H(x):=\prod_{i=0}^{\infty} P_{0}\left(x^{k^{i}}\right)
$$

converges to an invertible element of $K[[x]]$. By definition, $H(x)$ satisfies the following equation:

$$
H(x)=P_{0}(x) H\left(x^{k}\right)
$$


and hence $H(x)$ is a $k$-Becker power series. Now, set $G(x):=H(x) F(x)$. Then our assumption on $F(x)$ implies that

$$
\sum_{i=0}^{n} P_{i}(x) H\left(x^{k^{i}}\right)^{-1} G\left(x^{k^{i}}\right)=0 .
$$

Dividing both sides by $P_{0}(x) H(x)^{-1}$, we obtain that

$$
G(x)=-\sum_{i=1}^{n} P_{i}(x)\left(\prod_{j=1}^{i-1} P_{0}\left(x^{k^{i}}\right)\right) G\left(x^{k^{i}}\right) .
$$

This shows that $G$ is a $k$-Becker power series. Hence $F(x)$ can be written as

$$
F(x)=\left(\prod_{i=0}^{\infty} P_{0}\left(x^{k^{i}}\right)\right)^{-1} G(x),
$$

where $G(x)$ is a $k$-Becker power series. This ends the proof.

\section{Conditions on $k$ and $\ell$}

In this section, $K$ will denote an arbitrary field. We consider power series in $K[[x]]$ that are both $k$ - and $\ell$-Mahler with respect to two multiplicatively independent natural numbers $k$ and $\ell$. More specifically, we look at the set of natural numbers $m$ for which such a power series is necessarily $m$-Mahler.

Proposition 8.1. - Let $k$ and $\ell$ be two multiplicatively independent natural numbers and let $F(x) \in K[[x]]$ be a power series that is both $k$-and $\ell$-Mahler. Let us assume that $a$ and $b$ are integers with the property that $m:=k^{a} \ell^{b}$ is an integer greater than 1 . Then $F(x)$ is also $m$-Mahler.

Proof. - Let $V$ denote the $K(x)$-vector space spanned by all the power series that belong to the set $\left\{F\left(x^{k^{a} \ell^{b}}\right) \mid a, b \in \mathbb{N}\right\}$. By assumption, there exists some natural number $N$ such that $F\left(x^{k^{n}}\right) \in \sum_{i=0}^{N-1} K(x) F\left(x^{k^{i}}\right)$ and $F\left(x^{\ell^{n}}\right) \in \sum_{i=0}^{N-1} K(x) F\left(x^{\ell^{i}}\right)$ for every integer $n \geq N$. Thus $V$ is a $K(x)$ vector space of dimension at most $N^{2}$.

Suppose that $a$ and $b$ are integers such that $m:=k^{a} \ell^{b}$ is an integer greater than 1. If $a$ and $b$ are nonnegative, then $F\left(x^{m^{j}}\right) \in V$ for every integer $j \geq 0$ and since the dimension of $V$ is a finite, we see that $F(x)$ is $m$-Mahler. Thus we may assume that at least one of $a$ or $b$ is negative. Since $m \geq 1$, at least one of $a$ or $b$ must also be positive. Without loss of generality, we may thus assume that $a>0$ and $b<0$. 
We are now going to show that $F\left(x^{m^{j}}\right) \in V$ for every nonnegative integer $j$. To see this, we fix a nonnegative integer $j$. Then we observe that $m^{j} \ell^{-b j}=k^{j a}$ and thus $F\left(x^{m^{j} l^{i}}\right)$ belongs to $V$ for every integer $i \geq-b j$. Since $-b j \geq 0$, there exists a smallest nonnegative integer $i_{0}$ such that $F\left(x^{m^{j} \ell^{i}}\right) \in V$ for every integer $n \geq i_{0}$. If $i_{0}$ is zero, then we are done. We assume that $i_{0}$ is positive and look for a contradiction. By definition of $i_{0}$, we note that $F\left(x^{m^{j} \ell^{i} 0^{-1}}\right) \notin V$. By assumption, $F(x)$ satisfies a $\ell$-Mahler equation of the form

$$
\sum_{i=0}^{N} P_{i}(x) F\left(x^{\ell^{i}}\right)=0
$$

with $P_{0}(x), \ldots, P_{N}(x) \in K[x]$ and $P_{0}(x) \neq 0$. Applying the operator $x \mapsto$ $x^{m^{j} \ell^{i}{ }^{-1}}$, we get that

$$
P_{0}\left(x^{m^{j} \ell^{i}-1}\right) F\left(x^{m^{j} \ell^{i} 0-1}\right)=-\sum_{i=1}^{N} P_{i}\left(x^{m^{j} \ell^{i_{0}-1}}\right) F\left(x^{m^{j} \ell^{i_{0}-1+i}}\right) .
$$

By definition of $i_{0}$, the right-hand side of this equation is in $V$, and so $F\left(x^{m^{j} \ell^{i} 0^{-1}}\right) \in V$ since $P_{0}(x)$ is nonzero. This is a contradiction. It follows that $F\left(x^{m^{j}}\right) \in V$ for every nonnegative integer $j$.

Since $V$ is a $K(x)$-vector space of dimension at most $N^{2}$, we see that $F(x), F\left(x^{m}\right), \ldots, F\left(x^{m^{N^{2}}}\right)$ are linearly dependent over $K(x)$, which implies that $F(x)$ is $m$-Mahler. This ends the proof.

Corollary 8.1. - Let $k$ and $\ell$ be two multiplicatively independent natural numbers and let $F(x) \in K[[x]]$ be a power series that is both $k$-and $\ell$-Mahler. Then there exist two multiplicatively independent positive integers $k^{\prime}$ and $\ell^{\prime}$ such that the following conditions hold.

(i) There is a prime number $p$ that divides $k^{\prime}$ and does not divide $\ell^{\prime}$.

(ii) There is a prime number $q$ that divides $\ell^{\prime}$ and does not divide $k^{\prime}$.

(iii) $F(x)$ is both $k^{\prime}$ - and $\ell^{\prime}$-Mahler.

Proof. - There exist prime numbers $p_{1}, \ldots, p_{m}$ and nonnegative integers $a_{1}, \ldots, a_{m}, b_{1}, \ldots, b_{m}$ such that

$$
k=\prod_{i=1}^{m} p_{i}^{a_{i}} \text { and } \ell=\prod_{i=1}^{m} p_{i}^{b_{i}} .
$$

Moreover, we can assume that, for each $i$, at least one of $a_{i}$ or $b_{i}$ is positive.

Note that if there are $i$ and $j$ such that $a_{i}=0$ and $b_{j}=0$, then we can take $k^{\prime}:=k$ and $\ell^{\prime}:=\ell$ and set $p:=p_{j}$ and $q:=p_{i}$ to obtain the desired result. Thus 
we can assume without loss of generality that $b_{i}>0$ for $i \in\{1, \ldots, m\}$. Then there is some $i_{0} \in\{1, \ldots, m\}$ such that $a_{i_{0}} / b_{i_{0}} \leq a_{j} / b_{j}$ for all $j \in\{1, \ldots, m\}$. In particular, $c_{j}:=a_{j} b_{i_{0}}-b_{j} a_{i_{0}}$ is a nonnegative integer for all $j \in\{1, \ldots, m\}$. Hence

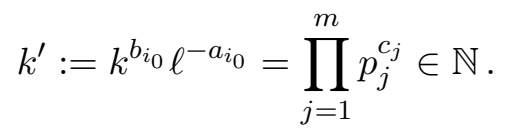

Furthermore, $p_{i_{0}}$ does not divide $k^{\prime}$ and since $k$ and $\ell$ are multiplicatively independent, the $c_{i}$ 's are not all equal to zero.

Now we pick $i_{1} \in\{1, \ldots, m\}$ such that $c_{i_{1}} / b_{i_{1}} \geq c_{j} / b_{j}$ for all $j \in\{1, \ldots, m\}$. Note that $c_{i_{1}}>0$ since the $c_{i}$ 's are not all equal to zero. Set

$$
\ell^{\prime}:=\ell^{c_{i_{1}}}\left(k^{\prime}\right)^{-b_{i_{1}}}=\prod_{j=1}^{m} p_{j}^{b_{j} c_{i_{1}}-b_{i_{1}} c_{j}} \in \mathbb{N} .
$$

Since $c_{i_{0}}=0, c_{i_{1}}>0$ and the $b_{i}$ 's are positive, we get that $p_{i_{0}}$ divides $\ell^{\prime}$. Moreover, $p_{i_{1}}$ does not divide $\ell^{\prime}$ while $p_{i_{1}}$ divides $k^{\prime}$ for $c_{i_{1}}$ is positive. In particular, $k^{\prime}$ and $\ell^{\prime}$ are multiplicatively independent. Furthermore, Proposition 8.1 implies that $F(x)$ is both $k^{\prime}$ - and $\ell^{\prime}$-Mahler. Setting $q:=p_{i_{0}}$ and $p=p_{i_{1}}$, we obtain that $k^{\prime}$ and $\ell^{\prime}$ have all the desired properties. This concludes the proof.

\section{Elimination of singularities at roots of unity}

In this section we look at the singularities of $k$-Mahler functions at roots of unity. Strictly speaking, we do not necessarily eliminate singularities, and so the section title is perhaps misleading. We do, however, show that one can reduce to the case of considering Mahler equations whose singularities at roots of unity have a restricted form.

Assumption-Notation 1. - Throughout this section we make the following assumptions and use the following notation.

(i) We assume that $k$ and $\ell$ are two multiplicatively independent natural numbers.

(ii) We assume there exist primes $p$ and $q$ such that $p \mid k$ and $p$ does not divide $\ell$ and such that $q \mid \ell$ and $q$ does not divide $k$.

(iii) We assume that $F(x)$ is a $k$-Mahler complex power series that satisfies an equation of the form

$$
\sum_{i=0}^{d} P_{i}(x) F\left(x^{k^{i}}\right)=0
$$

with $P_{0}, \ldots, P_{d} \in \mathbb{C}[x]$ and $P_{0}(0) \neq 0$. 
(iv) We assume that $F(x)$ is an $\ell$-Mahler complex power series that satisfies an equation of the form

$$
\sum_{i=0}^{e} Q_{i}(x) F\left(x^{\ell^{i}}\right)=0
$$

with $Q_{0}, \ldots, Q_{e} \in \mathbb{C}[x]$ and $Q_{0}(0) \neq 0$.

In this section, our aim is to prove the following result.

Theorem 9.1. - Let $F(x) \in \mathbb{C}[[x]]$ be a power series that satisfies Assumption-Notation 1 and that is not a polynomial. Then $F(x)$ satisfies a non-trivial $k$-Mahler equation of the form

$$
\sum_{i=0}^{d} P_{i}(x) F\left(x^{k^{i}}\right)=0
$$

with the property that $P_{0}(0)=1$ and $P_{0}(\alpha) \neq 0$ if $\alpha$ is a root of unity satisfying $\alpha^{k^{j}}=\alpha$ for some positive integer $j$.

9.1. Asymptotic estimates for some infinite products. - We first study the behaviour around the unit circle of infinite products of the form

$$
\left(\prod_{i=0}^{\infty} P\left(x^{k^{i}}\right)\right)^{-1}
$$

where $P(x) \in \mathbb{C}[x]$ and $P(0)=1$.

Lemma 9.1. - Let $k \geq 2$ be a natural number. Then

$$
\lim _{\substack{t \rightarrow 1 \\ 0<t<1}}\left(\prod_{j=0}^{\infty} \frac{1}{1-t^{k^{j}}}\right) \cdot(1-t)^{A}=\infty
$$

for every positive real number $A$. 
Proof. - Let $t$ be in $\left(1-1 / k^{9}, 1\right)$. Let $N \geq 2$ be the largest natural number such that $t \in\left(1-k^{-(N+1)^{2}}, 1\right)$. Then

$$
\begin{aligned}
\prod_{j=0}^{\infty}\left(1-t^{k^{j}}\right)^{-1} & \geq \prod_{j=0}^{N}\left(1-t^{k^{j}}\right)^{-1} \\
& =(1-t)^{-(N+1)} \prod_{j=0}^{N}\left(1+t+\cdots+t^{k^{j}-1}\right)^{-1} \\
& \geq(1-t)^{-(N+1)} \prod_{j=0}^{N} k^{-j} \\
& \geq(1-t)^{-(N+1)} k^{-(N+1)^{2}} \\
& >(1-t)^{-N} .
\end{aligned}
$$

By definition of $N$, we obtain that $t<1-k^{-(N+2)^{2}}$, which easily gives that

$$
N>\sqrt{\frac{-\log (1-t)}{4 \log k}} .
$$

This ends the proof for the right-hand side tends to infinity when $t$ tends to 1.

Lemma 9.2. - Let $k \geq 2$ be a natural number. Then for $t \in(0,1)$, we have

$$
\sum_{i=1}^{\infty} t^{i} / i \geq(1-1 / k) \sum_{i=0}^{\infty} t^{k^{i}} .
$$

Proof. - We have

$$
\begin{aligned}
\sum_{i=1}^{\infty} t^{i} / i & =t+\sum_{i=0}^{\infty} \sum_{j=k^{i}+1}^{k^{i+1}} t^{j} / j \\
& \geq t+\sum_{i=0}^{\infty} \sum_{j=k^{i}+1}^{k^{i+1}} t^{k^{i+1}} / k^{i+1} \\
& =t+\sum_{i=0}^{\infty} t^{k^{i+1}}\left(k^{i+1}-k^{i}\right) / k^{i+1} \\
& =t+(1-1 / k) \sum_{i=0}^{\infty} t^{k^{i+1}} \\
& \geq(1-1 / k) \sum_{i=0}^{\infty} t^{k^{i}},
\end{aligned}
$$


which ends the proof.

Lemma 9.3. - Let $k \geq 2$ be a natural number and let $\lambda \neq 1$ be a complex number. Then there exist two positive real numbers $A$ and $\varepsilon$ such that

$$
(1-t)^{A}<\left|\prod_{j=0}^{\infty} \frac{1}{1-\lambda t^{k^{j}}}\right|<(1-t)^{-A}
$$

whenever $1-\varepsilon<t<1$.

Proof. - We first prove the inequality on the right-hand side.

Note that since $\lambda \neq 1$ there exist two real numbers $\varepsilon_{0}$ and $c_{0}, c_{0}<1$, such that

$$
\inf \left\{\left|1-\lambda t^{k^{j}}\right| \mid t \in\left(1-\varepsilon_{0}, 1\right), j \geq 0\right\}>c_{0} .
$$

Let $t \in\left(1-\varepsilon_{0}, 1\right)$ and let $N$ be the largest nonnegative integer such that $t^{k^{N}} \geq 1 / 2$. Then for $j \geq 1$ we have $t^{k^{N+j}}=\left(t^{k^{N+1}}\right)^{k^{j-1}}<(1 / 2)^{k^{j-1}}$. Hence

$$
\left|1-\lambda t^{k^{N+j}}\right| \geq 1-|\lambda|(1 / 2)^{k^{j-1}} .
$$

Since the series $\sum_{j \geq 0}(1 / 2)^{k^{j-1}}$ converges, we get that the infinite product

$$
\prod_{j=0}^{\infty}\left|\frac{1}{1-\lambda t^{k^{N+j}}}\right|
$$

converges to some positive constant $c_{1}$. Then

$$
\begin{aligned}
\left|\prod_{j=0}^{\infty}\left(1-\lambda t^{k^{j}}\right)^{-1}\right| & =\prod_{j=0}^{N}\left|1-\lambda t^{k^{j}}\right|^{-1} \prod_{j=1}^{\infty}\left|1-\lambda t^{k^{N+j}}\right|^{-1} \\
& \leq\left(1 / c_{0}\right)^{N+1} c_{1} \\
& =\left(k^{N+1}\right)^{-\log c_{0} / \log k} c_{1} .
\end{aligned}
$$

Furthermore, we have by assumption that $t^{k^{N+1}}<1 / 2$ and thus $k^{N+1}<$ $-\log 2 / \log t$. This implies that

$$
\left|\prod_{j=0}^{\infty}\left(1-\lambda t^{k^{j}}\right)^{-1}\right| \leq c_{1}(-\log 2 / \log t)^{-\log c_{0} / \log k} .
$$

On the other hand, note that $\lim _{t \rightarrow 1}(1-t) / \log (t)=-1$ and hence there exists some positive $\varepsilon<\varepsilon_{0}$ such that

$$
c_{1}(-\log 2 / \log t)^{-\log c_{0} / \log k}<c_{1}(2 \log 2(1-t))^{\log c_{0} / \log k},
$$


whenever $t \in(1-\varepsilon, 1)$. Since $c_{0}<1$, we obtain that there exists a positive real number $A_{1}$ such that

$$
\left|\prod_{j=0}^{\infty}\left(1-\lambda t^{k^{j}}\right)^{-1}\right|<(1-t)^{-A_{1}}
$$

for all $t \in(1-\varepsilon, 1)$. This gives the right-hand side bound in the statement of the lemma.

To get the left-hand side, note that for all $t \in(0,1)$,

$$
\left|\prod_{j=0}^{\infty} \frac{1}{1-\lambda t^{k^{j}}}\right| \geq \prod_{j=0}^{\infty}\left(1+|\lambda| t^{k^{j}}\right)^{-1} \geq \prod_{j=0}^{\infty} \exp \left(-|\lambda| t^{k^{j}}\right) .
$$

By Lemma 9.2, we have

$$
\prod_{j=0}^{\infty} \exp \left(-|\lambda| t^{k^{j}}\right) \geq \exp \left(-|\lambda|(1-1 / k)^{-1} \sum_{i=1}^{\infty} t^{i} / i\right)=(1-t)^{|\lambda| k /(k-1)} .
$$

We thus obtain that, for all $t \in(0,1)$,

$$
\left|\prod_{j=0}^{\infty} \frac{1}{1-\lambda t^{k^{j}}}\right|>(1-t)^{A_{2}}
$$

where $A_{2}:=\lfloor|\lambda| k /(k-1)\rfloor+1$. Taking $A$ to be equal to the maximum of $A_{1}$ and $A_{2}$, we get the desired result.

Corollary 9.1. - Let $k \geq 2$ be a natural number, let $\alpha$ be root of unity that satisfies $\alpha^{k}=\alpha$, and let $P(x)$ be a nonzero polynomial with $P(0)=1$ and $P(\alpha) \neq 0$. Then there exist two positive real numbers $A$ and $\varepsilon>$ such that

$$
\left.(1-t)^{A}<\mid\left(\prod_{j=0}^{\infty} P\left((t \alpha)^{k^{j}}\right)\right)\right)^{-1} \mid<(1-t)^{-A}
$$

whenever $1-\varepsilon<t<1$.

Proof. - Let $\beta_{1}, \ldots, \beta_{s}$ denote the complex roots of $P$ (considered with muliplicities) so that we may factor $P(x)$ as $P(x)=\left(1-\beta_{1}^{-1} x\right) \cdots\left(1-\beta_{s}^{-1} x\right)$. We thus obtain

$$
\left|\prod_{j=0}^{\infty} \frac{1}{P\left((t \alpha)^{k^{j}}\right)}\right|=\prod_{i=1}^{s}\left|\prod_{j=0}^{\infty} \frac{1}{1-\beta_{i}^{-1} \alpha t^{k^{j}}}\right|,
$$


where $\beta_{i}^{-1} \alpha \neq 1$ for every $i \in\{1, \ldots, s\}$. Then by Lemma 9.3 , there are natural numbers $A_{i}$ and positive real numbers $\varepsilon_{i}, 0<\varepsilon_{i}<1$, such that

$$
(1-t)^{A_{i}}<\left|\prod_{j=0}^{\infty}\left(1-\beta_{i}^{-1} \alpha t\right)^{-1}\right|<(1-t)^{-A_{i}}
$$

whenever $1-\varepsilon_{i}<t<1$. Taking $\varepsilon:=\min \left(\varepsilon_{1}, \ldots, \varepsilon_{s}\right)$ and $A:=\sum_{i=1}^{s} A_{i}$, we obtain the desired result.

9.2. Asymptotic estimates for Becker functions. - We are now going to provide asymptotic estimates for Becker functions. We denote by $\|\cdot\|$ a norm on $\mathbb{C}^{d}$. We let $B(x, r)$ (respectively $\overline{B(x, r)}$ ) denote the open (respectively closed) ball of center $x$ and radius $r$. Our results will not depend on the choice of this norm.

Lemma 9.4. - Let $d$ and $k$ be two natural numbers, $\alpha$ a root of unity such that $\alpha^{k}=\alpha$, and $A: \overline{B(0,1)} \rightarrow M_{d}(\mathbb{C})$ a continuous matrix-valued function. Let us assume that $w(x) \in \mathbb{C}[[x]]^{d}$ satisfies the equation

$$
w(x)=A(x) w\left(x^{k}\right)
$$

for all $x \in B(0,1)$. Let us also assume that the following properties hold.

(i) The coordinates of $w(x)$ are analytic in $B(0,1)$ and continuous on $\overline{B(0,1)}$.

(ii) The matrix $A(\alpha)$ is not nilpotent.

(iii) The set $\{w(x) \mid x \in B(0,1)\}$ is not contained in a proper subspace of $\mathbb{C}^{d}$

Then there exist a positive real number $C$ and a subset $S \subseteq(0,1)$ that has 1 as a limit point such that

$$
\|w(t \alpha)\|>(1-t)^{C}
$$

for all $t \in S$.

Proof. - Since $A(\alpha)$ is not nilpotent, there is some natural number $e$ such that the kernel of $A(\alpha)^{e}$ and the kernel of $A(\alpha)^{e+1}$ are equal to a same proper subspace of $\mathbb{C}^{d}$, say $W$. Then there is a nonzero subspace $V$ such that $A(\alpha)(V) \subseteq V$ and $V \oplus W=\mathbb{C}^{d}$. Moreover, by compactness, there is a positive real number $c_{0}, c_{0}<1$, such that

$$
\|A(\alpha)(w)\| \geq c_{0}
$$

whenever $w \in V$ is a vector of norm 1 .

Since every vector has a unique decomposition as a sum of elements from $V$ and $W$, we have a continuous linear projection map $\pi: \mathbb{C}^{d} \rightarrow V$ with the 
property that $u-\pi(u) \in W$ for all $u \in \mathbb{C}^{d}$. We infer from Inequality (9.14) that

$$
\|\pi(A(\alpha)(u))\|=\|A(\alpha)(\pi(u))\| \geq c_{0}\|\pi(u)\|
$$

for all $u \in \mathbb{C}^{d}$. Since $A$ is continuous on $\overline{B(0,1)}$, Inequality (9.15) implies the existence of a positive constant $\varepsilon>0$ such that

$$
|\pi(A(x)(u))| \mid>c_{0}\|\pi(u)\| / 2,
$$

for all $u \in \mathbb{C}^{d}$ and all $x \in B(\alpha, \varepsilon) \cap \overline{B(0,1)}$. It follows by a simple induction that if $x_{1}, \ldots, x_{m} \in B(\alpha, \varepsilon) \cap \overline{B(0,1)}$ then

$$
|| \pi\left(A\left(x_{1}\right) \cdots A\left(x_{m}\right)(u)\right)\left|\geq\left(c_{0} / 2\right)^{m}\right| \mid \pi(u) \| .
$$

Set

$$
\mathcal{Z}:=\{t \in[0,1-\varepsilon / 2] \mid w(t \alpha) \in W)\} .
$$

We claim that $\mathcal{Z}$ is a finite set. Otherwise, there would be a nonzero row vector $u$ such that $u \cdot w(t \alpha)=0$ for infinitely many $t \in[0,1-\varepsilon / 2]$. But $u \cdot w(x)$ is analytic in $B(0,1)$ for $w(x)$ is and hence it would be identically zero on $B(0,1)$ by the identity theorem. This would contradict the assumption that $\{w(x) \mid x \in B(0,1)\}$ is not contained in a proper subspace of $\mathbb{C}^{d}$.

Let us pick a sequence $t_{0}, t_{1}, t_{2}, \ldots$ in $(0,1)$ such that:

- $t_{i}^{k}=t_{i-1}$ for $i \geq 1$;

- $t_{0} \in(1-\varepsilon, 1-\varepsilon / 2)$;

- $t_{0} \notin \mathcal{Z}$.

Note that $t_{n} \rightarrow 1$ as $n \rightarrow \infty$. Since $\mathcal{Z}$ is finite, there is an open neighbourhood $U \subseteq[0,1]$ of $\mathcal{Z}$ such that $t_{0} \notin U$. Set $X:=[0,1-\varepsilon / 2] \backslash U$. Then $X$ is compact and $\| \pi(w(x \alpha) \|$ is nonzero for $x \in X$. Thus there exists a positive real number $c_{1}$ such that

$$
\|\pi(w(x \alpha))\|>c_{1}
$$

for all $x \in X$. Then we infer from (9.16) that

$$
\begin{aligned}
\left\|\pi\left(w\left(t_{n} \alpha\right)\right)\right\| & =\| \pi\left(A\left(t_{n} \alpha\right) A\left(t_{n-1} \alpha\right) \cdots A\left(t_{1} \alpha\right)\left(w\left(t_{0} \alpha\right)\right) \|\right. \\
& \geq\left(c_{0} / 2\right)^{n} \| \pi\left(w\left(t_{0} \alpha\right) \|\right. \\
& >c_{1}\left(c_{0} / 2\right)^{n} .
\end{aligned}
$$

Furthermore, since the projection $\pi$ is continuous, there is some positive real number $c_{2}$ such that $\|\pi(u)\|<c_{2}\|u\|$ for all $u \in \mathbb{C}^{d}$. Thus

$$
\left\|w\left(t_{n}\right)\right\| \geq c_{2}^{-1}|| \pi\left(w\left(t_{n}\right)\right) \|>c_{2}^{-1} c_{1}\left(c_{0} / 2\right)^{n}
$$

for all $n \geq 1$. 
On the other hand, since

$$
\lim _{a \rightarrow 0^{+}} \frac{t_{0}^{a}-1}{a}=\log \left(t_{0}\right)<0,
$$

there exists some $\varepsilon_{0} \in(0,1)$ such that

$$
t_{0}^{a}<1+a \log \left(t_{0}\right) / 2<1-a\left(1-t_{0}\right) / 2
$$

for $a \in\left(0, \varepsilon_{0}\right)$. Thus if $n$ is large enough, say $n \geq n_{0}$, then $k^{n}>1 / \varepsilon_{0}$ and we have $t_{n}=\left(t_{0}\right)^{1 / k^{n}}<1-\left(1-t_{0}\right) /\left(2 k^{n}\right)$. Hence $k^{n}>\left(1-t_{0}\right) /\left(2\left(1-t_{n}\right)\right)$. Then we have

$$
\begin{aligned}
\left\|w\left(t_{n} \alpha\right)\right\| & >c_{2}^{-1} c_{1}\left(c_{0} / 2\right)^{n} \\
& =c_{2}^{-1} c_{1} k^{n \log _{k}\left(c_{0} / 2\right)} \\
& \geq\left(c_{2}^{-1} c_{1}\left(\frac{\left(1-t_{0}\right)}{2}\right)^{\log _{k}\left(c_{0} / 2\right)}\right)\left(1-t_{n}\right)^{-\log _{k}\left(c_{0} / 2\right)} .
\end{aligned}
$$

Thus if we take $C:=-2 \log _{k}\left(c_{0} / 2\right)>0$, the fact that $t_{n}$ tends to 1 as $n$ tends to infinity implies the existence of a positive integer $n_{1} \geq n_{0}$ such that

$$
\left\|w\left(t_{n} \alpha\right)\right\|>\left(1-t_{n}\right)^{C}
$$

for all $n \geq n_{1}$. Taking $S:=\left\{t_{n} \in(0,1) \mid n \geq n_{1}\right\}$, we obtain the desired result.

Lemma 9.5. - Let $B: \overline{B(0,1)} \rightarrow M_{d}(\mathbb{C})$ be a continuous matrix-valued function whose entries are analytic inside the unit disc and continuous on the closed unit disc. Let us assume that there exist two positive real numbers $\varepsilon$ and $M$ such that $|\operatorname{det}(B(x))|>(1-|x|)^{M}$ for every $x$ such that $1-\varepsilon<|x|<1$. Then there exists a positive real number $C$ such that for every column vector $u$ of norm 1, we have

$$
\| B(x)(u)|| \geq(1-|x|)^{C}
$$

for every $x$ such that $1-\varepsilon<|x|<1$.

Proof. - Our assumption implies that $B(x)$ is invertible for every $x$ such that $1-\varepsilon<|x|<1$. Let $\Delta(x)$ denote the determinant of $B(x)$. Using the classical adjoint formula for the inverse of $B(x)$, we see that $B(x)^{-1}$ has entries $c_{i, j}(x)$ that have the property that they are expressible (up to sign) as the ratio of the determinant of a submatrix of $B(x)$ and $\Delta(x)$. Since the entries of $B(x)$ are continuous on $\overline{B(0,1)}$, each determinant of a submatrix of $B(x)$ is also continuous. By compactness, we see that there is a positive real number $\kappa$ such that

$$
\left|c_{i, j}(x)\right| \leq \kappa /|\Delta(x)| \leq \kappa(1-|x|)^{-M}
$$


for every $(i, j) \in\{1, \ldots, d\}^{2}$ and every $x$ such that $1-\varepsilon<|x|<1$. Thus there exists a positive real number $C$ such that

$$
\left\|B(x)^{-1}\right\| \leq(1-|x|)^{-C}
$$

for every $x$ such that $1-\varepsilon<|x|<1$. It follows that if $u$ is a vector of norm 1 , then

$$
\|B(x)(u)\| \geq(1-|x|)^{C} .
$$

for every $x$ such that $1-\varepsilon<|x|<1$. The result follows.

Corollary 9.2. - Let $d$ and $k$ be two natural numbers, $\alpha$ be a root of unity such that $\alpha^{k}=\alpha, \zeta$ be a root on unity such that $\zeta^{k^{j}}=1$ for some natural number $j$, and $A: \overline{B(0,1)} \rightarrow M_{d}(\mathbb{C})$ be a continuous matrix-valued function. Let us assume that $w(x) \in \mathbb{C}[[x]]^{d}$ satisfies the equation

$$
w(x)=A(x) w\left(x^{k}\right)
$$

for all $x \in B(0,1)$. Let us also assume that the following properties hold.

(i) The coordinates of $w(x)$ are analytic in $B(0,1)$ and continuous on $\overline{B(0,1)}$.

(ii) The matrix $A(\alpha)$ is not nilpotent.

(ii) There exists two positive real numbers $\varepsilon$ and $M$ such that $|\operatorname{det}(A(x))|>$ $(1-|x|)^{M}$ for every $x$ with $1-\varepsilon<|x|<1$.

(iv) The set $\{w(x) \mid x \in B(0,1)\}$ is not contained in a proper subspace of $\mathbb{C}^{d}$.

Then there exist a positive real number $C$ and a subset $S \subseteq(0,1)$ that has 1 as a limit point such that

$$
\|w(t \alpha \beta)\|>(1-t)^{C}
$$

for all $t \in S$.

Proof. - Since $A(\alpha)$ is not nilpotent, we first infer from Lemma 9.4 that there exist a positive real number $C_{0}$ and a sequence $t_{n} \in(0,1)$, which tends to 1 , such that $\left\|w\left(t_{n} \alpha\right)\right\|>\left(1-t_{n}\right)^{C_{0}}$ for every integer $n \geq 1$. Let $s_{n} \in(0,1)$ be such that $s_{n}^{k^{j}}=t_{n}$. Then

$$
w\left(s_{n} \alpha \beta\right)=A\left(s_{n} \alpha \beta\right) A\left(s_{n}^{k} \alpha \beta^{k}\right) \cdots A\left(s_{n}^{k^{j-1}} \alpha \beta^{k^{j-1}}\right)\left(w\left(t_{n} \alpha\right)\right) .
$$

By assumption there exists a positive real number $M$ such that $|\operatorname{det}(A(x))|>$ $(1-|x|)^{M}$ for every $x$ with $1-\varepsilon<|x|<1$. Set

$$
B(x):=A(x \alpha \beta) A\left(x^{k} \alpha \beta^{k}\right) \cdots A\left(x^{k^{j-1}} \alpha \beta^{k^{j-1}}\right) .
$$


Then there is a positive real number $C_{1}$ such that if $(1-\varepsilon)^{1 / k^{j-1}}<|x|<1$ then

$$
\operatorname{det}(B(x))>(1-|x|)^{M} \cdots\left(1-|x|^{k^{j-1}}\right)^{M} \geq(1-|x|)^{j M} .
$$

It follows from Lemma 9.5 that there exists a positive real number $C_{1}$ such that for $n$ sufficiently large we have

$$
\begin{aligned}
\left\|w\left(s_{n} \alpha \beta\right)\right\| & =\left\|B\left(s_{n}\right)\left(w\left(t_{n} \alpha\right)\right)\right\|>\left(1-s_{n}\right)^{C_{1}}\left\|w\left(t_{n} \alpha\right)\right\| \\
& >\left(1-s_{n}\right)^{C_{1}}\left(1-t_{n}\right)^{C_{0}} .
\end{aligned}
$$

Since $\left(1-t_{n}\right) /\left(1-s_{n}\right) \rightarrow k^{j}$ as $n \rightarrow \infty$, we see that if we take $C:=2\left(C_{1}+C_{0}\right)$ then we have

$$
\left\|w\left(s_{n} \alpha \beta\right)\right\| \geq\left(1-s_{n}\right)^{C}
$$

for all $n$ sufficiently large. The result follows.

We are now almost ready to prove the main result of this section. Before doing this, we give the following simple lemma.

Lemma 9.6. - Let $d$ be a natural number and let $A$ be a $d \times d$ complex matrix whose $(i, j)$-entry is $\delta_{i, j+1}$ if $i \geq 2$. If there is an integer $r$ such that the $(1, r)$-entry of of $A$ is nonzero, then $A$ is not nilpotent.

Proof. - Let $\left(a_{1}, \ldots, a_{d}\right)$ denote the first row of $A$. Then by the theory of companion matrices, $A$ has characteristic polynomial $x^{d}-a_{1} x^{d-1}-a_{2} x^{d-2}-$ $\cdots-a_{d}$. But if $A$ is nilpotent, its characteristic polynomial must be $x^{d}$ and hence the first row of $A$ must be zero.

9.3. Proof of Theorem 9.1. — We are now ready to prove the main result of this section.

Proof of Theorem 9.1. - Consider the set $I$ of all polynomials $P(x) \in \mathbb{C}[x]$ for which there exist positive integers $a$ and $b$ with $a<b$ such that

$$
P(x) F(x) \in \sum_{i=a}^{b} \mathbb{C}[x] F\left(x^{k^{i}}\right) .
$$

We note that $I$ is an ideal of $\mathbb{C}[x]$. Let $P_{0}(x)$ be a generator for $I$. Let us assume that $\alpha$ is a root of $P_{0}(x)$ with the property that $\alpha^{k^{i}}=\alpha$ for some positive integer $i$. We will obtain a contradiction from this assumption.

Since $F(x)$ is $k$-Mahler, it is also $k^{i}$-Mahler and hence $F(x)$ satisfies a nontrivial polynomial equation

$$
\sum_{j=0}^{d} Q_{j}(x) F\left(x^{k^{i j}}\right)=0
$$


with $Q_{0}, \ldots, Q_{d}$ polynomials. We pick such a nontrivial relation with $Q_{0}$ nonzero and the degree of $Q_{0}$ minimal. By assumption $P_{0}$ divides $Q_{0}$ and so $\alpha$ is a root is of $Q_{0}(x)$. Also, we may assume that for some integer $j, 0<j \leq d$, we have $Q_{j}(\alpha) \neq 0$. Indeed, otherwise we could divide our equation by $(x-\alpha)$ to get a new relation with a new $Q_{0}$ of smaller degree.

By Lemma 6.1, there exists some natural number $N$ such that $F(x)$ can be decomposed as $F(x)=T(x)+x^{N} F_{0}(x)$, where $T(x)$ is a polynomial of degree $N-1$ and $F_{0}(x)$ is a power series with nonzero constant term such that $F_{0}(x)$ satisfies a $k^{i}$-Mahler equation

$$
\sum_{j=0}^{e} \widetilde{Q}_{j}(x) F_{0}\left(x^{k^{i j}}\right)=0
$$

with $\widetilde{Q}_{0}(0)=1, \widetilde{Q}_{0}(\alpha)=0$ and $\widetilde{Q}_{j}(\alpha) \neq 0$ for some integer $j, 0<j \leq e$. Moreover, by picking $N$ sufficiently large, we may assume that $F_{0}(x)$ satisfies a nontrivial $\ell$-Mahler equation

$$
\sum_{j=0}^{f} R_{j}(x) F_{0}\left(x^{\ell^{j}}\right)=0
$$

for some polynomials $R_{j}(x)$ with $R_{0}(0)=1$. Now, we infer from Proposition 7.4 that there is some $\ell$-Becker power series $G(x)$ such that

$$
F_{0}(x)=\left(\prod_{j=0}^{\infty} R_{0}\left(x^{\ell^{j}}\right)\right)^{-1} G(x) .
$$

For $i=0, \ldots, e$, we let $c_{i}$ denote the order of vanishing of $\widetilde{Q}_{i}(x)$ at $\alpha$, with the convention that $c_{i}=\infty$ if $\widetilde{Q}_{i}(x)=0$. We note that $0<c_{0}<\infty$ and that there is some $j, 0<j \leq e$, such that $c_{j}=0<c_{0}$. Let

$$
b:=\max \left\{\frac{c_{0}-c_{j}}{j} \mid j=1, \ldots, d\right\} .
$$

Since at least one of $c_{1}, \ldots, c_{d}$ is strictly less than $c_{0}$, we have that $b$ is positive. Moreover, by definition there is some $j_{0} \in\{1, \ldots, d\}$ such that $c_{j_{0}}+b j_{0}-c_{0}=0$. Then, for $j \in\{0, \ldots, d\}$, we set

$$
S_{j}(x):=\widetilde{Q}_{j}(x)\left(\prod_{n=0}^{j-1}\left(1-\alpha^{-1} x^{k^{i n}}\right)^{b}\right)\left(1-\alpha^{-1} x\right)^{-c_{0}} .
$$

Note that (9.19) implies that $S_{0}(x)$ is a polynomial in $\mathbb{C}[x]$ such that $S_{0}(0)=1$ and $S_{0}(\alpha) \neq 0$. 
Now, we set

$$
L(x):=F_{0}(x) \prod_{j=0}^{\infty} S_{0}\left(x^{k^{i j}}\right) \prod_{j=0}^{\infty}\left(1-\alpha^{-1} x^{k^{i j}}\right)^{b}
$$

and we infer from (9.18) that

$$
L(x) \prod_{j=0}^{\infty}\left(1-\alpha^{-1} x^{k^{i j}}\right)^{-b} \prod_{j=0}^{\infty} R_{0}\left(x^{\ell^{j}}\right)=G(x) \prod_{j=0}^{\infty} S_{0}\left(x^{k^{i j}}\right) .
$$

In the sequel, we are going to obtain some asymptotic estimates for the quantities $G(x), \prod_{j \geq 0} R_{0}\left(x^{\ell^{j}}\right), \prod_{j \geq 0} S_{0}\left(x^{k^{i j}}\right), L(x)$ and $\prod_{j \geq 0}\left(1-\alpha^{-1} x^{k^{i j}}\right)^{-b}$ in a neighbourhood of some root of unity. We will then show that these estimates are not compatible with Equality (9.22), providing the desired contradiction.

Estimate for $G(x)$. - Note first that, since $G(x)$ is a $\ell$-Becker power series, Theorem 7.2 implies that $G(x)$ is $\ell$-regular. By Proposition 7.2, there exist two positive real numbers $C$ and $m$ such that

$$
|G(x)|<C(1-|x|)^{-m},
$$

for even complex number $x$ in the open unit disc. This implies that there exist two positive real numbers $A_{0}$ and $\varepsilon_{0}$ such that

$$
|G(x)|<(1-|x|)^{-A_{0}}
$$

for every complex number $x$ with $1-\varepsilon_{0}<1-|x|<1$.

Asymptotic estimate for $\prod_{j \geq 0} R_{0}\left(x^{\ell^{j}}\right)$. - By assumption there is a prime $p$ that divides $k$ and does not divide $\ell$. Thus there exists some positive integer $N_{0}$ such that whenever $\zeta$ is a primitive $p^{n}$-th root of unity with $n \geq N_{0}$, then we have $R_{0}\left((\alpha \zeta)^{\ell^{j}}\right)$ is nonzero for every nonnegative integer $j$.

Let $\zeta$ be such a primitive $p^{n}$-th root of unity with $n \geq N_{0}$. Then there exist two positive integers $n_{1}$ and $n_{2}, n_{1}<n_{2}$, such that

$$
(\alpha \zeta)^{\ell^{n_{1}}}=(\alpha \zeta)^{\ell^{n_{2}}}
$$

Then for $t \in(0,1)$ we have

$$
\prod_{j=0}^{\infty} R_{0}\left((t \alpha \zeta)^{\ell^{j}}\right)=\prod_{j=0}^{n_{1}-1} R_{0}\left((t \alpha \zeta)^{\ell^{j}}\right) \prod_{i=n_{1}}^{n_{2}-1} \prod_{j=0}^{\infty} R_{0}\left(\left((t \alpha \zeta)^{\ell^{i}}\right)^{\ell^{j\left(n_{2}-n_{1}\right)}}\right) .
$$

Note that $\prod_{j=0}^{n_{1}-1} R_{0}(x)$ is a polynomial that does not vanish at any point of the finite set $\left.\left\{(\alpha \zeta)^{\ell^{j}}\right) \mid j \geq 0\right\}$. This gives that there exist two positive real 
numbers $\delta$ and $\varepsilon_{1}$ such that

$$
\left.\mid \prod_{j=0}^{n_{1}-1} R_{0}(t \alpha \zeta)^{\ell^{j}}\right) \mid>\delta
$$

for all $t \in\left(1-\varepsilon_{1}, 1\right)$. Furthermore, Equality (9.24) implies that for every integer $i, n_{1} \leq i \leq n_{2}-1$, we have

$$
\left((\alpha \zeta)^{\ell^{i}}\right)^{\ell^{j\left(n_{2}-n_{1}\right)}}=\left((\alpha \zeta)^{\ell^{i}}\right) .
$$

Thus, for every integer $i, n_{1} \leq i \leq n_{2}-1$, we can apply Corollary 9.1 to the infinite product

$$
\prod_{j=0}^{\infty} R_{0}\left(\left((t \alpha \zeta)^{\ell^{i}}\right)^{\ell^{j\left(n_{2}-n_{1}\right)}}\right) .
$$

This finally implies the existence of a positive real number $\varepsilon_{2}=\varepsilon_{2}(\zeta)$ and a positive integer $A_{1}=A_{1}(\zeta)$ such that

$$
\left|\prod_{j=0}^{\infty} R_{0}\left((t \alpha \zeta)^{\ell^{j}}\right)\right|>(1-t)^{A_{1}}
$$

for $t \in\left(1-\varepsilon_{2}, 1\right)$.

Asymptotic estimate for $\prod_{j \geq 0} S_{0}\left(x^{k^{j}}\right)$. - First note that since $\alpha^{k}=\alpha$, $S_{0}(0)=1$ and $\alpha$ is not a root of $S_{0}$, we can apply Corollary 9.1. We thus obtain the existence of a positive real number $\delta_{0}$ and a positive integer $M_{0}$ such that

$$
\left|\prod_{j=0}^{\infty} S_{0}\left((t \alpha)^{k^{i j}}\right)\right|<(1-t)^{M_{0}}
$$

for every $t \in\left(1-\delta_{0}, 1\right)$.

Now, if $\zeta$ is a primitive $p^{n}$-th root of unity, for some positive integer $n$, we have $(\alpha \zeta)^{k^{i j}}=\alpha$ for all $j \geq n$. This implies that

$$
\prod_{j=0}^{\infty} S_{0}\left((t \alpha \zeta)^{k^{i j}}\right)=R(t) \prod_{j=0}^{\infty} S_{0}\left((t \alpha)^{k^{i j}}\right),
$$

where

$$
R(t)=\left(\prod_{j=0}^{n-1} S_{0}\left((t \alpha \zeta)^{k^{i j}}\right)\right)\left(\prod_{j=0}^{n-1} S_{0}\left((t \alpha)^{k^{i j}}\right)\right)^{-1}
$$


Since $\alpha^{k^{i j}}=\alpha$ and $S_{0}(\alpha) \neq 0$, there are two positive real number $\delta_{1}$ and $C$ such that

$$
|R(t)|<C
$$

for every $t \in\left(1-\delta_{1}, 1\right)$. We thus infer from (9.26), (9.27) and (9.28) that there exist a positive real number $\varepsilon_{3}=\varepsilon_{3}(\zeta)$ and a positive integer $A_{2}=A_{2}(\zeta)$ such that

$$
\left|\prod_{j=0}^{\infty} S_{0}\left((t \alpha \zeta)^{\ell^{j}}\right)\right|<(1-t)^{-A_{2}}
$$

for $t \in\left(1-\varepsilon_{3}, 1\right)$.

Asymptotic estimate for $L(x)$. - We first infer from (9.17) and (9.21) that the function $L$ satisfies the following relation:

$$
\sum_{n=0}^{e} \widetilde{Q}_{n}(x)\left(\prod_{j=n}^{\infty} S_{0}\left(x^{k^{i j}}\right)^{-1}\right)\left(\prod_{j=n}^{\infty}\left(1-\alpha^{-1} x^{k^{i j}}\right)^{-b}\right) L\left(x^{k^{i n}}\right)=0,
$$

which gives by $(9.20)$ that

$$
\begin{aligned}
L(x) & =-\sum_{n=1}^{e}\left(\widetilde{Q}_{n}(x) \widetilde{Q}_{0}(x)^{-1} \prod_{j=0}^{n-1} S_{0}\left(x^{k^{i j}}\right) \prod_{j=0}^{n-1}\left(1-\alpha^{-1} x^{k^{i j}}\right)^{b}\right) L\left(x^{k^{i n}}\right) \\
& =\left(\widetilde{Q}_{n}(x)\left(\prod_{j=0}^{n-1}\left(1-\alpha^{-1} x^{k^{i j}}\right)^{b}\right)\left(1-\alpha^{-1} x\right)^{-c_{0}} S_{0}(x)^{-1} \prod_{j=0}^{n-1} S_{0}\left(x^{k^{i j}}\right)\right) L\left(x^{k^{i n}}\right) \\
& =-\sum_{n=1}^{e}\left(S_{n}(x) \prod_{j=1}^{n-1} S_{0}\left(x^{k^{i j}}\right)\right) L\left(x^{k^{i n}}\right) .
\end{aligned}
$$

Let $A(x)$ denote the $e \times e$ matrix whose $(i, j)$-entry is $\delta_{i, j+1}$ if $i \geq 2$ and whose $(1, j)$-entry is

$$
C_{j}(x):=-S_{n}(x) \prod_{j=1}^{n-1} S_{0}\left(x^{k^{i j}}\right)
$$

for $j=1, \ldots, e$. Then the previous computation gives us the following functional equation:

$$
\left[L(x), L\left(x^{k^{i}}\right), \ldots, L\left(x^{k^{i(e-1)}}\right)\right]^{T}=A(x)\left[L\left(x^{k^{i}}\right), \ldots, L\left(x^{k^{i e}}\right)\right]^{T} .
$$


We claim that if $\zeta$ is a primitive $p^{n}$-th root of unity with $n \geq N_{0}+i(e-$ 1) $\nu_{p}(k)$, then there exist a positive integer $M_{0}=M_{0}(\zeta)$ and an infinite sequence $\left(t_{n}\right)_{n \geq 0} \in(0,1)^{\mathbb{N}}$ which tends to 1 such that

$$
\left\|\left[L\left(t_{n} \alpha \zeta\right), L\left(t_{n}^{k^{i}} \alpha \zeta^{k^{i}}\right), \ldots, L\left(t_{n}^{k^{i(e-1)}} \alpha \zeta^{k^{i(e-1)}}\right)\right]^{T}\right\|>\left(1-t_{n}\right)^{M_{0}} .
$$

In order to obtain Inequality (9.31) it remains to prove that we can apply Corollary 9.2 to $L(x)$. Note that $L(x)$ is not identically zero since $F(x)$ is not a polynomial. Furthermore, we can assume that $L$ is not a nonzero constant since otherwise Inequality (9.31) would be immediately satisfied.

(i) By definition, $S_{n}(x)=\widetilde{Q}_{n}(x)\left(\prod_{j=0}^{n-1}\left(1-\alpha^{-1} x^{k^{i j}}\right)^{b}\right)\left(1-\alpha^{-1} x\right)^{-c_{0}}$. Moreover, a simple computation gives that $\prod_{j=0}^{n-1}\left(1-\alpha^{-1} x^{k^{i j}}\right)^{b}=(1-$ $\left.\alpha^{-1} x\right)^{b n} P_{n}(x)^{b}$, for some polynomial $P_{n}(x)$ that does not vanish at $\alpha$. By definition of $c_{n}$, this shows that

$$
S_{n}(x)=\left(1-\alpha^{-1} x\right)^{c_{n}+b n-c_{0}} P_{n}(x)^{b} R_{n}(x),
$$

where $P_{n}(x)$ and $R_{n}(x)$ are two polynomials that do not vanish at $\alpha$. By definition of $b$, we have $c_{n}+b n-c_{0} \geq 0$ for $n \in\{0, \ldots, e\}$, and thus $S_{n}(x)$ is analytic in the open unit disc and continuous on the closed unit disc. Since the finite product $\prod_{j=1}^{n-1} S_{0}\left(x^{k^{i j}}\right)$ is a polynomial, this shows that the entries of the matrix $A(x)$ are analytic on $B(0,1)$ and continuous on $\overline{B(0,1)}$.

(ii) The definition of $b$ implies that there is some integer $r, 1 \leq r \leq e$, such that $c_{r}+b r-c_{0}=0$. Since $P_{r}(\alpha) R_{r}(\alpha) \neq 0$, Equation (9.32) implies that $S_{r}(\alpha) \neq 0$. On the other hand, we have that $\prod_{j=0}^{r-1} S_{0}\left(x^{k^{i j}}\right)$ does not vanish at $\alpha$ since $S_{0}(\alpha) \neq 0$ and $\alpha^{k^{i}}=\alpha$. We thus obtain that the $(1, r)$-entry of $A(\alpha)$ is nonzero. By Lemma 9.6, this implies that $A(\alpha)$ is not nilpotent.

(iii) By definition of the matrix $A$, we get that

$$
\operatorname{det} A(x)=(-1)^{e} C_{e}(x)=(-1)^{e+1} S_{e}(x) \prod_{n=1}^{e-1} S_{0}\left(x^{k^{i n}}\right) .
$$

By (9.32), we have that $S_{e}(x)=\left(1-\alpha^{-1} x\right)^{c_{e}+b e-c_{0}} P_{e}(x)^{b} R_{e}(x)$, where $P_{e}(x)$ and $R_{e}(x)$ are polynomials. It follows that there exist two positive real numbers $\delta$ and $M$ such that

$$
|\operatorname{det} A(x)|>(1-|x|)^{M}
$$

for every $x$ such that $1-\delta<|x|<1$. 
(iv) We claim that

$$
\left\{\left[L(x), L\left(x^{k^{i}}\right), \ldots, L\left(x^{k^{i(e-1)}}\right)\right]^{T} \mid x \in B(0,1)\right\}
$$

cannot be contained in a proper subspace of $\mathbb{C}^{e}$. Indeed, if it were, then there would exist some nonzero row vector $u$ such that

$$
u\left[L(x), L\left(x^{k^{i}}\right), \ldots, L\left(x^{k^{i(e-1)}}\right)\right]^{T}=0
$$

for all $x \in B(0,1)$. But this would give that $L(x), \ldots, L\left(x^{k^{i(e-1)}}\right)$ are linearly dependent over $\mathbb{C}$, and hence by Lemma 7.1 , we would obtain that $L(x)$ is a constant function, a contradiction.

It follows from (i), (ii), (iii) and (iv) that we can apply Corollary 9.2 to $L(x)$, which proves that (9.31) holds. Then, we deduce from (9.31) that there exist a sequence $\left(s_{n}\right)_{n \geq 0}$ in $(0,1)$ which tends to 1 , some root of unity $\mu$ that has order at least $p^{N_{0}}$ and some positive integer $A_{3}=A_{3}(\zeta)$ such that

$$
\left|L\left(s_{n} \alpha \mu\right)\right|>\left(1-s_{n}\right)^{A_{3}}
$$

for every positive integer $n$.

Conclusion. - By Equation (9.22), we have

$$
\begin{aligned}
& \left|L\left(s_{n} \alpha \mu\right) \prod_{j=0}^{\infty}\left(1-\alpha^{-1}\left(s_{n} \mu\right)^{k^{i j}}\right)^{-b} \prod_{j=0}^{\infty} R_{0}\left(\left(s_{n} \alpha \mu\right)^{\ell^{j}}\right)\right| \\
& =\left|G\left(s_{n} \alpha \mu\right) \prod_{j=0}^{\infty} S_{0}\left(\alpha\left(s_{n} \mu\right)^{k^{i j}}\right)\right| .
\end{aligned}
$$

By Equations (9.23) and (9.29), we see that the right-hand side is at most

$$
\left(1-s_{n}\right)^{-\left(A_{0}+A_{2}\right)}
$$

for every integer $n$ large enough. Similarly, by Equations (9.25) and (9.33), the left-hand side is at least

$$
\left(1-s_{n}\right)^{A_{1}+A_{3}} \prod_{j=0}^{\infty}\left(1-\alpha^{-1}\left(s_{n} \mu\right)^{k^{i j}}\right)^{-b}
$$

for every integer $n$ large enough. Thus we have

$$
\left|\prod_{j=0}^{\infty}\left(1-\alpha^{-1}\left(s_{n} \mu\right)^{k^{i j}}\right)^{-b}\right|<\left(1-s_{n}\right)^{-\left(A_{0}+A_{1}+A_{2}+A_{3}\right)}
$$

for every integer $n$ large enough. But this contradicts Lemma 9.1, since $\mu^{k^{j}}=1$ for all sufficiently large $j$. This concludes the proof. 


\section{Existence of prime ideals with special properties}

In this section we prove the following result.

Theorem 10.1. - Let $R$ be a principal localization of a number ring and let $P(x), Q(x) \in R[x]$ be two polynomials with $P(0)=Q(0)=1$ and such that none of the zeros of $P(x) Q(x)$ are roots of unity. Then there are infinitely many prime ideals $\mathfrak{P}$ in $R$ such that

$$
\left(\prod_{i=0}^{\infty} P\left(x^{k^{i}}\right)\right)^{-1} \bmod \mathfrak{P}
$$

is a $k$-automatic power series in $(R / \mathfrak{P})[[x]]$ and

$$
\left(\prod_{i=0}^{\infty} Q\left(x^{\ell^{i}}\right)\right)^{-1} \bmod \mathfrak{P}
$$

is a $\ell$-automatic power series in $(R / \mathfrak{P})[[x]]$.

Our proof is based on Chebotarev's density theorem for which we refer the reader for example to $[\mathbf{2 1}]$ and to the informative survey $[\mathbf{2 2}]$. We first prove a basic lemma about non-existence of $n$-th roots of elements in a number field for sufficiently large $n$. The proof makes use of the notion of Weil absolute logarithmic height. We do not recall the precise definition of Weil height, as it is a bit long and not really within the scope of the present paper. However, we are only going to use basic properties of this height that can be found in any standard book such as $[\mathbf{1 9}],[\mathbf{2 0}]$, or $[\mathbf{4 0}]$.

Lemma 10.1. - Let $K$ be a number field and let $\alpha$ be a nonzero element in $K$ that is not a root of unity. Then for all sufficiently large natural numbers $n$ the equation $\beta^{n}=\alpha$ has no solution $\beta \in K$.

Proof. - This result is an easy consequence of the theory of heights. Given $x \in K$, we denote by $h(x)$ the Weil absolute logarithmic height of $x$.

Since $K$ is a number field, it has the Northcott property, that is for every positive real number $M$ the set $\{x \in K \mid h(x) \leq M\}$ is finite. In particular, there exists a positive real number $\varepsilon$ depending only on $K$ such that if $h(x)<\varepsilon$ then $h(x)=0$. Let $n$ be an integer such that $n>h(\alpha) / \varepsilon$. Let us assume that there is $\beta \in K$ such that $\beta^{n}=\alpha$. Since $h\left(x^{k}\right)=k h(x)$ for every $x \in K$ and $k \in \mathbb{N}$, we obtain that $h(\beta)=h(\alpha) / n<\varepsilon$. Thus $h(\beta)=0$. By Kronecker's theorem, this implies that $\beta$ is a root of unity and thus $\alpha$ is also be a root of unity, a contradiction. 
Lemma 10.2. - Let $m$ be a natural number and let $d_{1}, \ldots, d_{m}$ be positive integers. Suppose that $H$ is a subgroup of

$$
\prod_{i=1}^{m}\left(\mathbb{Z} / d_{i} \mathbb{Z}\right)
$$

with the property that there exist natural numbers $r_{1}, \ldots, r_{m}$ with

$$
1 / r_{1}+\cdots+1 / r_{m}<1
$$

such that for each $i \in\{1, \ldots, m\}$, there is an element $h_{i} \in H$ whose $i$-th coordinate has order $r_{i}$. Then there is an element $h \in H$ such that no coordinate of $h$ is equal to zero.

Proof. - For each $i \in\{1, \ldots, m\}$, we let

$$
\pi_{i}: \prod_{i=1}^{m}\left(\mathbb{Z} / d_{i} \mathbb{Z}\right) \rightarrow \mathbb{Z} / d_{i} \mathbb{Z}
$$

denote the projection onto the $i$-th coordinate. Given $\left(x_{1}, \ldots, x_{m}\right) \in \mathbb{Z}^{m}$ we have that $x_{1} h_{1}+\cdots+x_{m} h_{m} \in H$. Observe that the density of integers $y$ for which

$$
\pi_{i}\left(\sum_{j \neq i} x_{j} h_{j}+y h_{i}\right)=0
$$

is equal to $1 / r_{i}$. Since this holds for all $\left(x_{1}, x_{2}, \ldots, x_{i-1}, x_{i+1}, \ldots, x_{m}\right) \in \mathbb{Z}^{m-1}$, we see that the density of $\left(x_{1}, \ldots, x_{m}\right) \in \mathbb{Z}^{m}$ for which

$$
\pi_{i}\left(\sum_{j=1}^{m} x_{j} h_{j}\right)=0
$$

is equal to $1 / r_{i}$. Thus the density of $\left(x_{1}, \ldots, x_{m}\right) \in \mathbb{Z}^{m}$ for which

$$
\pi_{i}\left(\sum_{j=1}^{m} x_{j} h_{j}\right)=0
$$

holds for some $i \in\{1, \ldots, m\}$ is at most

$$
1 / r_{1}+\cdots+1 / r_{m}<1 .
$$

In particular, we see that there is some $\left(x_{1}, \ldots, x_{m}\right) \in \mathbb{Z}^{m}$ such that the element $h:=x_{1} h_{1}+\cdots+x_{m} h_{m} \in H$ has no coordinate equal to zero.

Lemma 10.3. - Let $k \geq 2$ be an integer, let $R$ be a principal localization of a number ring, let $\mathfrak{P}$ be a nonzero prime ideal of $R$, and let a be an element of 
R. Suppose that for some natural number $n$, the polynomial $1-a x^{k^{n}} \bmod \mathfrak{P}$ has no roots in $R / \mathfrak{P}$. Then the infinite product

$$
\left(\prod_{j=0}^{\infty}\left(1-a x^{k^{j}}\right)\right)^{-1} \bmod \mathfrak{P}
$$

is a $k$-automatic power series in $(R / \mathfrak{P})[[x]]$.

Proof. - Set $F(x):=\prod_{j=0}^{\infty}\left(1-a x^{k^{j}}\right)^{-1} \bmod \mathfrak{P}$. Without loss of generality we can assume that $a$ does not belong to $\mathfrak{P}$. Let us first note that the sequence $a, a^{k}, a^{k^{2}}, \ldots$ is necessarily eventually periodic modulo $\mathfrak{P}$. However, it cannot be periodic, as otherwise the polynomial $1-a x^{k^{n}}$ would have a root for every natural number $n$. Thus there exists a positive integer $N$ such that

$$
a \not \equiv a^{k^{N}} \equiv a^{k^{2 N}} \bmod \mathfrak{P} .
$$

Set $b:=a^{k^{N}}$ and let us consider the polynomial

$$
Q(x):=(1-b x)\left(1-b x^{k}\right) \cdots\left(1-b x^{k^{N-1}}\right) .
$$

Now arguing exactly as in the proof of Proposition 7.3, we see that there exist polynomial $S(x) \in R[x]$ such that $G(x):=Q(x)^{-1} F(x)$ satisfies the equation

$$
G(x) \equiv S(x) G\left(x^{k}\right) \bmod \mathfrak{P} .
$$

Thus Theorem 5.3 implies that $G(x) \bmod \mathfrak{P}$ is a $k$-regular power series in $(R / \mathfrak{P})[[x]]$. By Proposition 7.1 , we see that $F(x) \bmod \mathfrak{P}$ is a $k$-regular power series since it is a product of a polynomial (which is $k$-regular) and a $k$-regular power series. Since the base field is finite, Proposition 7.1 gives that $F(x) \bmod$ $\mathfrak{P}$ is actually a $k$-automatic power series. This ends the proof.

Proof of Theorem 10.1. - By assumption $R$ is a principal localization of a number field $K$. Let $L$ be the Galois extension of $K$ generated by all complex roots of the polynomial $P(x) Q(x)$. Thus there are $\alpha_{1}, \ldots, \alpha_{d}, \beta_{1}, \ldots, \beta_{e} \in L$ such that $P(x)=\left(1-\alpha_{1} x\right) \cdots\left(1-\alpha_{d} x\right)$ and $Q(x)=\left(1-\beta_{1} x\right) \cdots\left(1-\beta_{e} x\right)$. We fix a prime $p$ that divides $k$ and a prime $q$ that divides $\ell$. Let $s$ be a natural number such that $p^{s}$ and $q^{s}$ are both larger than $d+e$. Since by assumption none of the roots of $P(x) Q(x)$ is a root of unity, Lemma 10.1 implies that, for $1 \leq i \leq d$ and $1 \leq j \leq e$, there are largest nonnegative integers $n_{i}$ and $m_{j}$ with the property that we can write $\alpha_{i}=\gamma_{i}^{p^{n_{i}}} u_{i}$ and $\beta_{j}=\delta_{j}^{q^{m_{j}}} v_{j}$ for some elements $\gamma_{i}, \delta_{j} \in L\left(e^{2 \pi i /\left(p^{s} q^{s}\right)}\right)$ and $u_{i}, v_{j}$ roots of unity in $L\left(e^{2 \pi i /\left(p^{s} q^{s}\right)}\right)$.

Next let $n$ denote a natural number that is strictly larger than the maximum of the $n_{i}$ and the $m_{j}$ for $1 \leq i \leq d$ and $1 \leq j \leq e$. Set $E:=L\left(e^{2 \pi i /\left(p^{n} q^{n}\right)}\right)$ and 
let $F$ denote the Galois extension of $E$ generated by all complex roots of the polynomial

$$
\prod_{i=1}^{d} \prod_{j=1}^{e}\left(x^{p^{n}}-\gamma_{i}\right)\left(x^{q^{n}}-\delta_{j}\right) .
$$

For each $i, 1 \leq i \leq d$, we pick a root $\gamma_{i, 0}$ of $x^{p^{n}}-\gamma_{i}$, and for each $j, 1 \leq j \leq e$, we pick a root $\delta_{j, 0}$ of $x^{q^{n}}-\delta_{j}$.

Claim. We claim that for every integer $i, 1 \leq i \leq d$, there is an automorphism $\sigma_{i}$ in $\operatorname{Gal}(F / E)$ such that

$$
\sigma_{i}\left(\gamma_{i, 0}\right)=\gamma_{i, 0} u
$$

with $u$ a primitive $p^{r}$-th root of unity for some $r$ greater than or equal to $s$. Similarly, for every integer $j, 1 \leq j \leq e$, there is an automorphism $\tau_{j}$ in $\operatorname{Gal}(F / E)$ that such that

$$
\tau_{j}\left(\delta_{j, 0}\right)=\gamma_{j, 0} u^{\prime},
$$

for some primitive $q^{r^{\prime}}$-th root of unity $u^{\prime}$ with $r^{\prime}$ greater than or equal to $s$.

Proof of the claim. Note that

$$
\left\{\frac{\sigma\left(\gamma_{i, 0}\right)}{\gamma_{i, 0}} \mid \sigma \in \operatorname{Gal}(F / E)\right\}
$$

forms a subgroup of the $p^{n}$-th roots of unity. To prove the claim we just have to prove that this group cannot be contained in the group of $p^{s-1}$-st roots of unity. Let us assume that this is the case. Then the product of the Galois conjugates of $\gamma_{i, 0}$ must be $\tilde{\gamma}_{i}:=\gamma_{i, 0}^{p^{t}} v$ for some $t<s$ and some root of unity $v$. Moreover, $\tilde{\gamma}_{i}$ lies in $L\left(e^{2 \pi i /\left(p^{n} q^{n}\right)}\right)$. Note that the Galois group of $L\left(e^{2 \pi i /\left(p^{n} q^{n}\right)}\right)$ over $L\left(e^{2 \pi i /\left(p^{s} q^{s}\right)}\right)$ has order dividing $\phi\left(p^{n} q^{n}\right) / \phi\left(p^{s} q^{s}\right)=p^{n-s} q^{n-s}$. Since all conjugates of $\tilde{\gamma}_{i}$ are equal to $\tilde{\gamma}_{i}$ times some root of unity, we see that the relative norm of $\tilde{\gamma}_{i}$ with respect to the subfield $L\left(e^{2 \pi i /\left(p^{s} q^{s}\right)}\right)$ is of the form $\tilde{\gamma}_{i}^{d} v^{\prime}$ for some divisor $d$ of $p^{n-s} q^{n-s}$ and some root of unity $v^{\prime}$. Moreover,

$$
\tilde{\gamma}_{i}^{d} v^{\prime} \in L\left(e^{2 \pi i /\left(p^{s} q^{s}\right)}\right) \text {. }
$$

Note that the gcd of $d$ and $p^{n-t}$ is equal to $p^{n-s_{0}}$ for some integer $s_{0} \geq s$. Since $\gamma_{i, 0}^{p^{n}}=\tilde{\gamma}_{i}{ }^{n-t} v^{-p^{n-t}} \in L\left(e^{2 \pi i /\left(p^{s} q^{s}\right)}\right)$, we see by expressing $p^{n-s_{0}}$ as an integer linear combination of $d$ and $p^{n-t}$ that

$$
\tilde{\gamma}_{i}{ }^{n-s_{0}} \omega=\gamma_{i, 0}^{p^{n-s_{0}+t}} \omega^{\prime} \in L\left(e^{2 \pi i /\left(p^{s} q^{s}\right)}\right)
$$

for some roots of unity $\omega$ and $\omega^{\prime}$ and some $s_{0} \geq s$. But $s_{0}-t \geq 1$ and so we see that $\alpha_{i}$ is equal to a root of unity times

$$
\left(\gamma_{i, 0}^{p^{n-s_{0}+t}} \omega^{\prime}\right)^{p^{s_{0}-t+n_{i}}}
$$

contradicting the maximality of $n_{i}$. This confirms the claim. 
For an integer $m$, we let $\mathbb{U}_{m}$ denote the subgroup of $\mathbb{C}^{*}$ consisting of all $m$-th roots of unity. Note that we can define a group homomorhpism $\Phi$ from $\operatorname{Gal}(F / E)$ to $\left(\mathbb{U}_{p^{n}}\right)^{d} \times\left(\mathbb{U}_{q^{n}}\right)^{e}$ by

$$
\Phi(\sigma):=\left(\sigma\left(\gamma_{1,0}\right) / \gamma_{1,0}, \ldots, \sigma\left(\gamma_{d, 0}\right) / \gamma_{d, 0}, \sigma\left(\delta_{1,0}\right) / \delta_{1,0}, \ldots, \sigma\left(\delta_{e, 0}\right) / \delta_{e, 0}\right) .
$$

We see that $\Phi$ is a group homomorphism since each $\sigma \in \operatorname{Gal}(F / E)$ fixes the $p^{n}$-th and $q^{n}$-th roots of unity. Set $H:=\Phi(\operatorname{Gal}(F / E))$. The claim implies that the $i$-th coordinate in $\left(\mathbb{U}_{p^{n}}\right)^{d}$ of $\Phi\left(\sigma_{i}\right)$ has order at least equal to $p^{s}$. Similarly, it also implies that the $j$-th coordinate in $\left(\mathbb{U}_{q^{n}}\right)^{e}$ of $\Phi\left(\tau_{j}\right)$ has order at least equal to $q^{s}$. Since $p^{s}$ and $q^{s}$ are both greater than $d+e$, we have

$$
d / p^{s}+e / q^{s}<1 .
$$

Now, since $\left(\mathbb{U}_{p^{n}}\right)^{d} \times\left(\mathbb{U}_{q^{n}}\right)^{e} \cong\left(\mathbb{Z} / p^{n} \mathbb{Z}\right)^{d} \times\left(\mathbb{Z} / q^{n} \mathbb{Z}\right)^{e}$, we infer from Lemma 10.2 that there exists an element $h$ in $H$ such that every coordinate of $h$ is different from the identity element. In other words, this means that there exists some element $\tau$ of $\operatorname{Gal}(F / E)$ that fixes no element in the set

$$
\left\{\gamma_{i, 0} \mid 1 \leq i \leq d\right\} \cup\left\{\delta_{j, 0} \mid 1 \leq j \leq e\right\} .
$$

Since by definition $\tau$ fixes all $p^{n}$-th and $q^{n}$-th roots of unity, we see more generally that no root of the polynomial

$$
\prod_{i=1}^{d} \prod_{j=1}^{e}\left(x^{p^{n}}-\gamma_{i}\right)\left(x^{q^{n}}-\delta_{j}\right)
$$

is fixed by $\tau$. Since $\tau$ belongs to $\operatorname{Gal}(F / E)$, we can see $\tau$ as an element of $\operatorname{Gal}(F / K)$ that fixes all elements of $E$. We have thus produce an element $\tau$ of $\operatorname{Gal}(F / K)$ that fixes all roots of $P(x) Q(x)$ but that that does not fix any of the roots of the polynomial

$$
\prod_{i=1}^{d} \prod_{j=1}^{e}\left(x^{p^{n}}-\gamma_{i}\right)\left(x^{q^{n}}-\delta_{j}\right) .
$$

It follows from Chebotarev's density theorem (see for instance the discussion in [22]) that there is an infinite set of nonzero prime ideals $\mathcal{S} \subseteq \operatorname{Spec}(R)$ such that if $\mathfrak{P} \in \mathcal{S}$ then $P(x) Q(x) \bmod \mathfrak{P}$ factors into linear terms while the minimal polynomial of

$$
\prod_{i=1}^{d} \prod_{j=1}^{e}\left(x^{p^{n}}-\gamma_{i}\right)\left(x^{q^{n}}-\delta_{j}\right)
$$

over $K$ has no root modulo $\mathfrak{P}$. In particular, there is a natural number $N$ larger than $n$ such that for all such prime ideals $\mathfrak{P}$, the polynomial $P(x) Q(x) \bmod \mathfrak{P}$ splits into linear factors, while the polynomial $P\left(x^{p^{N}}\right) Q\left(x^{q^{N}}\right) \bmod \mathfrak{P}$ does not have any roots in $R / \mathfrak{P}$. 
For such a prime ideal $\mathfrak{P}$, there thus exist $a_{1}, \ldots, a_{d}, b_{1}, \ldots, b_{e}$ in the finite field $R / \mathfrak{P}$ such that

$$
P(x) \equiv\left(1-a_{1} x\right) \cdots\left(1-a_{d} x\right) \bmod \mathfrak{P}
$$

and

$$
Q(x) \equiv\left(1-b_{1} x\right) \cdots\left(1-b_{d} x\right) \bmod \mathfrak{P}
$$

Then

$$
\left(\prod_{j=0}^{\infty} P\left(x^{k^{j}}\right)\right)^{-1} \equiv \prod_{i=1}^{d}\left(\prod_{j=0}^{\infty}\left(1-a_{i} x^{k^{j}}\right)\right)^{-1} \bmod \mathfrak{P}
$$

By Lemma 10.3 the right-hand side is a product of $k$-automatic power series and hence, by Proposition 7.1, is $k$-automatic. Thus the infinite product

$$
\left(\prod_{j=0}^{\infty} P\left(x^{k^{j}}\right)\right)^{-1} \bmod \mathfrak{P}
$$

is a $k$-automatic power series in $R / \mathfrak{P}[[x]]$. Similarly, we get that

$$
\left(\prod_{j=0}^{\infty} Q\left(x^{\ell^{j}}\right)\right)^{-1} \equiv \prod_{i=1}^{e}\left(\prod_{j=0}^{\infty}\left(1-b_{i} x^{\ell^{j}}\right)\right)^{-1} \bmod \mathfrak{P},
$$

which implies that the infinite product

$$
\left(\prod_{j=0}^{\infty} Q\left(x^{\ell^{j}}\right)\right)^{-1} \bmod \mathfrak{P}
$$

is a $\ell$ automatic power series in $R / \mathfrak{P}[[x]]$. This concludes the proof.

\section{Proof of Theorem 1.1}

We are now ready to prove our main result.

Proof of Theorem 1.1. - Let $K$ be a field of characteristic zero and $k$ and $l$ be two multiplicatively independent positive integers.

We first note that if $F(x) \in K[[x]]$ is a rational function, then for every integer $m \geq 2$, it obviously satisfies a functional equation as in (1.3) with $n=0$. Hence, $F(x)$ is $m$-Mahler, which gives a first implication.

To prove the converse implication, we fix $F(x) \in K[[x]]$ that is both $k$ - and $\ell$-Mahler and we aim at proving that $F(x)$ is a rational function. Of course, if $F(x)$ is a polynomial, there is nothing to prove. From now on, we thus assume that $F(x)$ is not a polynomial. By Corollary 8.1, we can assume that there are primes $p$ and $q$ such that $p$ divides $k$ while $p$ does not divide $q$ and such 
that $q$ divides $\ell$ while $q$ does not divide $k$. By Theorem 5.1, we can assume that there is a ring $R$ that is a principal localization of a number ring such that $F(x) \in R[[x]]$ and satisfies the equations

$$
\sum_{i=0}^{n} P_{i}(x) F\left(x^{k^{i}}\right)=0
$$

with $P_{0}, \ldots, P_{d} \in R[x]$ and

$$
\sum_{i=0}^{m} Q_{i}(x) F\left(x^{\ell^{i}}\right)=0
$$

with $Q_{0}, \ldots, Q_{e} \in R[x]$. Without loss of generality, we can assume that all complex roots of $P_{0}(x)$ and $Q_{0}(x)$ belong to $R$ (otherwise we could just enlarge $R$ by adding these numbers). Furthermore, we can assume that $P_{0}(x) Q_{0}(x) \neq$ 0 . By Corollary 6.1, we can also assume that $P_{0}(0)=1$ and that $Q_{0}(0)=1$, for otherwise we could just replace $F(x)$ by the power series $F_{0}(x)$ given there. We choose a ring embedding of $R$ in $\mathbb{C}$ and for the moment we regard $F(x)$ as a complex power series. By Theorem 9.1, we can assume that if $\alpha$ is a root of unity such that $\alpha^{k^{j}}=\alpha$ for some positive integer $j$, then $P_{0}(\alpha) \neq 0$. Similarly, we can assume that if $\beta$ is a root of unity such that $\beta^{\ell^{j}}=\beta$ for some positive integer $j$, then $Q_{0}(\beta) \neq 0$.

By Proposition 7.4, we can write

$$
F(x)=\left(\prod_{j=0}^{\infty} P_{0}\left(x^{k^{j}}\right)\right)^{-1} G(x),
$$

for some $k$-regular power series $G(x) \in R[[x]]$. Furthermore, we can decompose $P_{0}(x)$ as $P_{0}(x)=S_{0}(x) S_{1}(x)$, where $S_{0}(x)$ and $S_{1}(x)$ are two polynomials, the zeros of $S_{0}(x)$ are all roots of unity, none of the zeros of $S_{1}(x)$ are roots of unity, and $S_{0}(0)=S_{1}(0)=1$. Since by assumption all roots of $P_{0}(x)$ lie in $R$, we get that both $S_{0}(x)$ and $S_{1}(x)$ belong to $R[x]$. By assumption if $\alpha$ is a root of $S_{0}(x)$ then for every positive integer $j$, one has $\alpha^{k^{j}} \neq \alpha$. Then, it follows from Proposition 7.3 that

$$
\left(\prod_{j=0}^{\infty} S_{0}\left(x^{k^{j}}\right)\right)^{-1} \in R[[x]]
$$


is a $k$-regular power series. Set $H:=\prod_{j=0}^{\infty} S_{0}\left(x^{k^{j}}\right)^{-1} G(x)$. We infer from Proposition 7.1 that $H(x)$ is a $k$-regular power series. Moreover, one has

$$
F(x)=\left(\prod_{j=0}^{\infty} S_{1}\left(x^{k^{j}}\right)\right)^{-1} H(x)
$$

Similarly, by Proposition 7.4, we can write

$$
F(x)=\left(\prod_{j=0}^{\infty} Q_{0}\left(x^{k^{j}}\right)\right)^{-1} I(x)
$$

for some $k$-regular power series $I(x) \in R[[x]]$. As previously, we can decompose $Q_{0}(x)$ as $Q_{0}(x)=T_{0}(x) T_{1}(x)$, where $T_{0}(x)$ and $T_{1}(x)$ belong to $R[x]$, the zeros of $T_{0}(x)$ are all roots of unity, none of the zeros of $T_{1}(x)$ are roots of unity, and $T_{0}(0)=T_{1}(0)=1$. By assumption if $\beta$ is a root of $T_{0}(x)$ then for every positive integer $j$, one has $\beta^{\ell^{j}} \neq \beta$. Then it follows from Proposition 7.3 that

$$
\left(\prod_{j=0}^{\infty} T_{0}\left(x^{\ell^{j}}\right)\right)^{-1} \in R[[x]]
$$

is a $\ell$-regular power series. Set $J:=\prod_{j=0}^{\infty} T_{0}\left(x^{k^{j}}\right)^{-1} I(x)$. Again, we see by Proposition 7.1 that $J(x)$ is $\ell$-regular. Moreover, one has

$$
F(x)=\left(\prod_{j=0}^{\infty} T_{1}\left(x^{k^{j}}\right)\right)^{-1} J(x) .
$$

By Theorem 10.1, there is an infinite set of nonzero prime ideals $\mathcal{S}$ of $R$ such that, for every prime ideal $\mathfrak{P}$ in $\mathcal{S}$,

$$
\left(\prod_{j=0}^{\infty} S_{1}\left(x^{k^{j}}\right)\right)^{-1} \bmod \mathfrak{P}
$$

is a $k$-automatic power series in $(R / \mathfrak{P})[[x]]$ and

$$
\left(\prod_{j=0}^{\infty} T_{1}\left(x^{\ell^{j}}\right)\right)^{-1} \bmod \mathfrak{P}
$$

is a $\ell$-automatic power series in $(R / \mathfrak{P})[[x]]$. Then we infer from Equalities (11.34) and (11.35) that, for $\mathfrak{P} \in \mathcal{S}, F(x) \bmod \mathfrak{P}$ is $k$-regular for it is the 
product of two $k$-regular power series. Similarly, $F(x) \bmod \mathfrak{P}$ is a $\ell$-regular power series.

We recall that the principal localization of a number ring is a Dedekind domain; that is, it is a noetherian normal domain of Krull dimension one. In particular, all nonzero prime ideals are maximal. Now since $R$ is a finitely generated $\mathbb{Z}$-algebra and $\mathfrak{P}$ is a maximal ideal, the quotient ring $R / \mathfrak{P}$ is a finite field (see [15, Theorem 4.19, p. 132]). By Proposition 7.1, this implies that $F(x) \bmod \mathfrak{P}$ is actually both $k$ - and $\ell$-automatic. By Cobham's theorem, we obtain that the sequence of coefficients of $F(x) \bmod \mathfrak{P}$ is eventually periodic and hence $F(x) \bmod \mathfrak{P}$ is a rational function.

Note that since $\mathcal{S}$ is infinite, the intersection of all ideals in $\mathcal{S}$ is the zero ideal (see [15, Lemma 4.16, p. 130]). Moreover, $F(x) \bmod \mathfrak{P}$ is rational for every prime ideal $\mathfrak{P} \in \mathcal{S}$. Applying Lemma 5.3 , we obtain that $F(x)$ is a rational function. This ends the proof.

Acknowledgement. - The authors would like to thank Michel Mendès France for his comments and encouragements. The first author is indebted to Éric Delaygue for his help with Maple. He is also most grateful to Macha and Vadim for inspiring discussions all along the preparation of this paper.

\section{References}

[1] B. Adamczewski and Y. Bugeaud, On the complexity of algebraic numbers I. Expansions in integer bases, Ann. of Math. 165 (2007), 547-565.

[2] B. Adamczewski and Y. Bugeaud, Real and p-adic expansions involving symmetric patterns, Int. Math. Res. Not., Volume 2006 (2006), Article ID 75968, 17 pages.

[3] B. Adamczewski and J. P. Bell, Diagonalization and rationalization of algebraic Laurent series, to appear in Ann. Sci. Éc. Norm. Supér., 2013.

[4] J.-P. Allouche and J. Shallit, Automatic sequences. Theory, applications, generalizations, Cambridge University Press, Cambridge, 2003.

[5] J.-P. Allouche and J. Shallit, The ring of k-regular sequences, Theoret. Comput. Sci. 98 (1992), 163-197.

[6] J.-P. Allouche and J. Shallit, The ring of k-regular sequences, II, Theoret. Comput. Sci. 307 (2003), 3-29.

[7] P.-G. Becker, k-Regular power series and Mahler-type functional equations, J. Number Theory 49 (1994), 269-286.

[8] J. P. Bell, A generalization of Cobham's theorem for regular sequences, Sém. Lothar. Combin. 54A (2005/07), Art. B54Ap, 15 pp. 
[9] A. Cobham, On the Hartmanis-Stearns problem for a class of tag machines, Conference Record of 1968 Ninth Annual Symposium on Switching and Automata Theory, Schenectady, New York (1968), 51-60.

[10] A. Cobham, On the base-dependence of sets of numbers recognizable by finite automata, Math. Systems Theory 3 (1969), 186-192.

[11] Ph. Dumas, Récurrences mahlériennes, suites automatiques, études asymptotiques, Thèse de doctorat, Universite Bordeaux I, Talence, 1993.

[12] Ph. Dumas and Ph. Flajolet, Asymptotique des récurrences mahlériennes: le cas cyclotomique, J. Théor. Nombres Bordeaux 8 (1996), 1-30.

[13] F. Durand, Cobham's theorem for substitutions, J. Eur. Math. Soc. 13 (2011), 1799-1814.

[14] S. Eilenberg, Automata, Languages, and Machines, Vol. A. Academic Press, 1974.

[15] D. Eisenbud, Commutative algebra. With a view toward algebraic geometry, Graduate Texts in Mathematics 150 Springer-Verlag, New York, 1995.

[16] G. Everest, A. van der Poorten, I. Shparlinski and T. Ward, Recurrence Sequences, Mathematical Surveys and Monographs 104, American Mathematical Society, Providence, RI, 2003.

[17] H. Furstenberg, Disjointness in ergodic theory, minimal sets, and a problem in Diophantine approximation, Math. Systems Theory 1 (1967), 1-49.

[18] J. Hartmanis and R. E. Stearns, On the computational complexity of algorithms, Trans. Amer. Math. Soc. 117 (1965), 285-306.

[19] M. Hindry and J. H. Silverman, Diophantine geometry, Graduate Texts in Mathematics 201, Springer-Verlag, New York, 2000.

[20] S. Lang, Fundamentals of Diophantine geometry, Springer-Verlag, New York, 1983.

[21] S. Lang, Algebraic number theory, Graduate Texts in Mathematics 110, SpringerVerlag, New York, 1994.

[22] H. W. Lenstra and P. Stevenhagen, Chebotarëv and his density theorem, Math. Intelligencer 18 (1996), 26-37.

[23] J. H. Loxton, Automata and transcendence in New advances in transcendence theory (Durham 1986), Cambridge University Press (1988), 215-228.

[24] J. H. Loxton and A. J. van der Poorten, Arithmetic properties of the solutions of a class of functional equations, J. reine angew. Math. 330 (1982), 159-172.

[25] J. H. Loxton and A. J. van der Poorten, Arithmetic properties of automata: regular sequences, J. reine angew. Math. 392 (1988), 57-610.

[26] K. Mahler, Arithmetische Eigenschaften der Lösungen einer Klasse von Funktionalgleichungen, Math. Ann. 101 (1929), 342-367.

[27] K. Mahler, Arithmetische Eigenschaften einer Klasse transzendentaltranszendente Funktionen, Math. Z. 32 (1930), 545-585.

[28] K. Mahler, Über das Verschwinden von Potenzreihen mehrerer Veränderlichen in speziellen Punktfolgen, Math. Ann. 103 (1930), 573-587. 
[29] K. Mahler, Some suggestions for further research, Bull. Austral. Math. Soc. 29 (1984), 101-108.

[30] M. Mendès France, Nombres algébriques et théorie des automates, Enseign. Math. 26 (1980), 193-199.

[31] Ku. Nishioka, New approach in Mahler's method, J. reine angew. Math. 407 (1990), 202-219.

[32] Ku. Nishioka, Algebraic independence measures of the values of Mahler's functions, J. reine angew. Math. 420 (1991), 203-214.

[33] Ku. Nishioka, Algebraic independence by Mahler's method and S-unit equations, Compos. Math. 92 (1994), 87-110.

[34] Ku. Nishioka, Mahler functions and transcendence, Lecture Notes in Math. 1631, Springer-Verlag, Berlin, 1997.

[35] F. Pellarin, Aspects de l'indépendance algébrique en caractéristique non nulle, Astérisque 317 (2008) 205-242 (2008). Séminaire Bourbaki. Vol. 2006/2007, Exp. No. 973.

[36] F. Pellarin, An introduction to Mahler's method for transcendence and algebraic independence,to appear in the EMS proceedings of the conference "Hodge structures, transcendence and other motivic aspects".

[37] A. J. van der Poorten, On the transcendence and algebraic independence of certain somewhat amusing numbers (results of Loxton and Van der Poorten), Séminaire de Théorie des Nombres 1975-1976 (Univ. Bordeaux I, Talence), Exp. No. $14,13 \mathrm{pp}$.

[38] A. J. van der Poorten, Remarks on automata, functional equations and transcendence, Séminaire de Théorie des Nombres de Bordeaux (1986-1987), Exp. No. 27, 11pp.

[39] B. Randé, Récurrence 2- et 3-mahlériennes, J. Théor. Nombres Bordeaux 5 (1993), 101-109.

[40] M. Waldschmidt, Diophantine approximation on linear algebraic groups, Grundlehren der Mathematischen Wissenschaften 326, Springer-Verlag, Berlin, 2000.

[41] M. Waldschmidt, Un demi-siècle de transcendance. In: Development of mathematics 1950-2000, pp. 1121-1186, Birkhäuser, Basel, 2000.

[42] D. Zagier, Solution to advanced problem 6625, Amer. Math. Monthly 99 (1992), $66-69$.

[43] U. Zannier, On a functional equation relating a Laurent series $f(x)$ to $f\left(x^{m}\right)$, Aequationes Math. 55 (1998), 15-43.

Boris Adamczewski, CNRS, Université de Lyon, Université Lyon 1, Institut Camille Jordan, 43 boulevard du 11 novembre 1918, 69622 Villeurbanne Cedex, France

E-mail : Boris. Adamczewski@math.univ-lyon1.fr

Jason P. Bell, Department of Pure Mathematics, University of Waterloo, Waterloo, ON, Canada, N2L 3G1 • E-mail : jpbell@uwaterloo.ca 